\title{
Chloroplast Hsp70 Isoform Is Required for Age-Dependent Tissue Preference of Bamboo mosaic virus in Mature Nicotiana benthamiana Leaves
}

\author{
Ying Wen Huang, ${ }^{1}$ Chung Chi Hu, ${ }^{1}$ Ching Hsiu Tsai, ${ }^{1}$ Na Sheng Lin, ${ }^{1,2}$ and Yau Heiu Hsu ${ }^{1}$ \\ ${ }^{1}$ Graduate Institute of Biotechnology, National Chung Hsing University, Taichung 40227, Taiwan; and ${ }^{2}$ Institute of Plant and \\ Microbial Biology, Academia Sinica, Taipei 11529, Taiwan \\ Accepted 27 April 2017.
}

\begin{abstract}
Plant viruses may exhibit age-dependent tissue preference in their hosts but the underlying mechanisms are not well understood. In this study, we provide several lines of evidence to reveal the determining role of a protein of the Nicotiana benthamiana chloroplast Hsp70 (NbcpHsp70) family, NbcpHsp70-2, involved in the preference of Bamboo mosaic virus (BaMV) to infect older tissues. NbcpHsp70 family proteins were identified in complexes pulled down with BaMV replicase as the bait. Among the isoforms of NbcpHsp70, only the specific silencing of NbcpHsp70-2 resulted in the significant decrease of BaMV RNA in $N$. benthamiana protopalsts, indicating that NbcpHsp70-2 is involved in the efficient replication of BaMV RNA. We further identified the age-dependent import regulation signal contained in the transit peptide of NbcpHsp70-2. Deletion, overexpression, and substitution experiments revealed that the signal in the transit peptide of NbcpHsp70-2 is crucial for both the import of NbcpHsp70-2 into older chloroplasts and the preference of BaMV for infecting older leaves of $N$. benthamiana. Together, these data demonstrated that BaMV may exploit a cellular agedependent transportation mechanism to target a suitable environment for viral replication.
\end{abstract}

Tissue tropism is an important subject in virology research because it provides vital information on why and how some viruses prefer certain tissues which, in turn, may be used for developing strategies against viral infection.

The important issue of age-related tissue tropism of plant viruses has been relatively neglected by virologists. However, understanding why and how some viruses show age-dependent tissue preferences would shed light on the underlying mechanisms governing important aspects of viral infection cycles such as replication or movement. Age-related resistance has been reported for bacterial pathogens in Arabidopsis thaliana and other plants (Carella et al. 2015; Develey-Rivière and Galiana 2007; Kus et al. 2002). For plant viruses, many years ago, Nicotiana tabacum varieties showed leaf position- and age-dependent susceptibility to Tobacco mosaic virus (TMV) (Takahashi 1972). The underlying mechanism was revealed by recent studies

Corresponding author: Y. H. Hsu; Telephone: +1 886-4-2285-6468; Fax: +1 886-4-2285-6468; E-mail: yhhsu@nchu.edu.tw

*The $\boldsymbol{e}$-Xtra logo stands for "electronic extra" and indicates that one supplementary method, one supplementary table, and three supplementary figures are published online.

(C) 2017 The American Phytopathological Society demonstrating that the replicase of TMV could age dependently interact with phloem-specific auxin/indole acetic acid transcription regulators which, in turn, modulates the transcriptional responses to enhance phloem loading and systemic spreading of TMV in mature leaves (Collum et al. 2016). In addition, age-dependent resistance against viral infection and affecting variation in viral content in younger and older tissues have been reported for a few other plant viruses, including Potato virus X (PVX) (Venekamp and Beemster 1980), Potato virus Y (PVY) (Venekamp et al. 1980), and Barley yellow dwarf virus-PAV strain (Sadeghi et al. 2000), and attributed to the decrease in ribosome and RNA content in older leaves (for PVX) or unspecified mechanisms. However, whether other plant viruses also exhibit age-dependent tissue preferences and their host factor mechanisms remain largely unknown.

Because of limited viral genome sizes, plant viruses heavily depend on host factors in their infection cycles and often have to improvise with the housekeeping genes of the hosts (Huang et al. 2012a; Nagy and Pogany 2012). Many host factors have been found involved in various stages of viral infection cycles, especially in viral replication complexes (VRC) from replication stages (Hyodo and Okuno 2014; Mine et al. 2010; Nagy and Pogany 2012; Nagy et al. 2016; Nishikiori et al. 2006). However, the involvement of these host factors in age-dependent tissue preferences still awaits further exploration.

Bamboo mosaic virus (BaMV) is a well-studied species in the genus Potexvirus, family Alphaflexiviridae. It has a singlestranded positive-sense RNA (ss[+]RNA) genome with the typical structure features and genome organization of potexviruses (Fig. 1A) (Lin et al. 1994; Yang et al. 1997). The genome contains five open reading frames that encode five proteins: replication protein (Rep) $(155 \mathrm{kDa})$, triple-gene-block protein 1 (TGBp1) (28 kDa), TGBp2 (13 kDa), TGBp3 (6 kDa), and capsid protein (CP) (25 kDa). An in vitro RNA-dependent RNA polymerase (RdRp) assay system has been established for BaMV to analyze the components of host and viral proteins associated with VRC (Cheng et al. 2001; Huang et al. 2008). UV-induced cross-linking analyses with the $3^{\prime}$ untranslated region (3'UTR) of BaMV genomic RNA (gRNA) used as a probe revealed several BaMV 3'UTR-interacting proteins, including elongation factor 1a, chloroplast phosphoglycerate kinase (PGK), glyceraldehyde 3-phosphate dehydrogenase, and heat shock protein 90 homolog (Hsp90) (Cheng et al. 2013; Huang et al. 2012b; Lin et al. 2007; Prasanth et al. 2011). Among these host factors, Hsp family members (Hsps) have been widely implicated in the infection cycles of many plant and animal viruses (Nagy et al. 2011; Park and Seo 2015). During virus infection, a large amount of viral proteins produced in a short period of time usually induces stress 
and then upregulates the expression of Hsps (Alam and Rochon 2016; Nagy et al. 2011). Therefore, many viruses exploit Hsps for various functions in their infection cycles such as replication, intracellular transport, and virion assembly (Verchot 2012). The cytosolic molecular chaperone Hsp70 is frequently recruited by many viruses (Dufresne et al. 2008; Mayer 2005; Pogany et al. 2008; Weeks and Miller 2008). In addition to cytosolic Hsps, plastid-targeted Hsp70 (cpHsp70) has been shown to participate in geminivirus Abutilon mosaic virus (AbMV) intra- and intercellular trafficking of nucleoprotein complexes via an interaction between cpHsp70 and AbMV movement protein (MP) (Krenz et al. 2010).

A. thaliana has 14 genes encoding members of the Hsp70 family: two of them (AtcpHsp70-1 and AtcpHsp70-2) encode

A

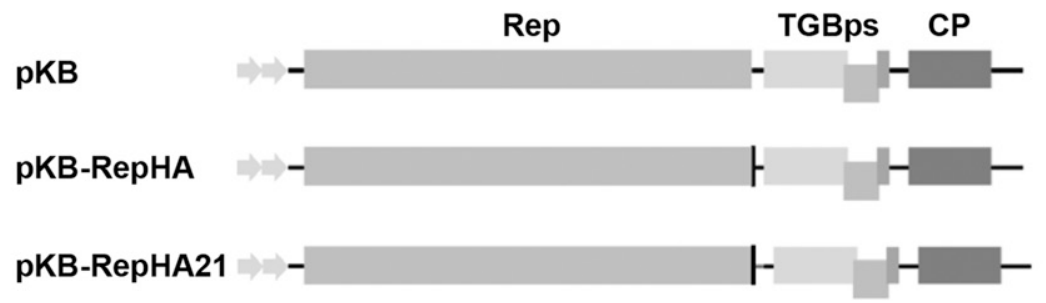

Upstream of Triple Gene Block (TGB)

BaMV

CUAGUUAAGUAACCUAUUAGUUUA ${ }^{*}-10$ nt- AUG

BaMV-RepHA CUAGUUAUCCAUACGACGUUCCAGAUUACGCAUAAGUAACCUAUUAGUUUÄ -10nt- AUG

BaMV-RepHA21 CUAGUUAUCCAUACGACGUUCCAGAUUACGCAUAAGAAgGUUUAUUCUCUAGUUAAGUAACCUAUUAGUUUA -10nt- AUG $21 \mathrm{nt}$

B

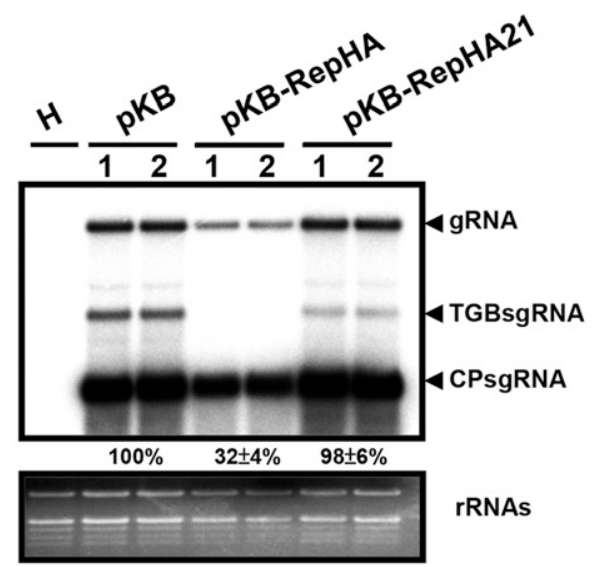

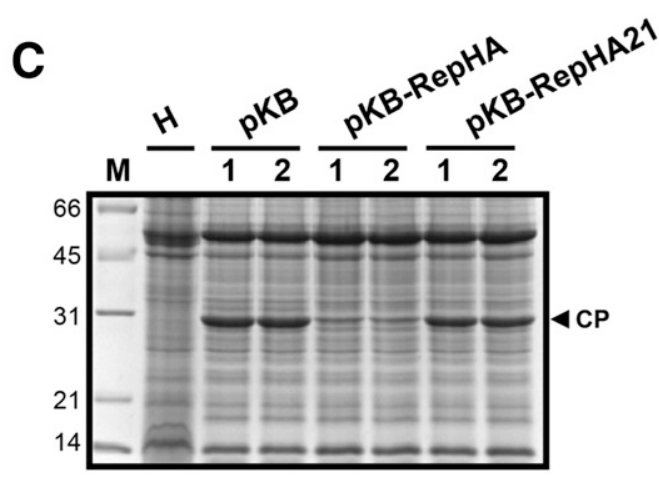

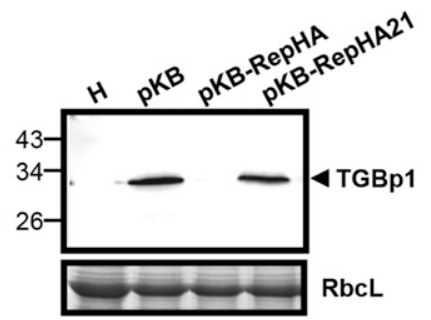

Fig. 1. Construction and characterization of Bamboo mosaic virus (BaMV)-expressing replication protein (Rep) with a C-terminal HA fusion. A, Schematic representations of genome organization of BaMV $(\mathrm{pKB})$ and BaMV expressing Rep-HA (pKB-RepHA and pKB-RepHA21) driven by a dual 35S promoter (gray arrow). Five open reading frames of BaMV encode Rep, triple gene block (TGB)p1-3 (TGBps), and capsid protein (CP), respectively. The plasmid pKBRepHA was generated from pKB by insertion of the HA sequence, shown as the black box, at $3^{\prime}$ end of the replicase coding region. Additional gray bar after HA in pKB-RepHA21 indicates the 21-nucleotide insertion for restoring the putative TGB subgenomic RNA (sgRNA) promoter sequence. Shown sequences are the putative promoter region for TGBsgRNA transcription that locates upstream to TGB of BaMV, BaMV-RepHA, and BaMV-RepHA21, respectively. Proposed conserved octanucleotide motif is shown in bold, and the asterisk indicates the transcriptional start site (Lee et al. 2000). The stop codon of replicase gene UAA is underlined. AUG denotes initiation codon for the TGBp1. The HA sequence is shown in the gray box. B, Northern blot analysis of the accumulation of BaMV genomic RNA (gRNA), sgRNA for TGBp1 (TGBsgRNA), and sgRNA for CP (CPsgRNA) in Nicotiana benthamiana plants individually infiltrated with pKB, pKB-RepHA, and pKB-RepHA21 for 4 days. Lane $\mathrm{H}$ represents healthy plant without BaMV infection as a negative control. Numbers on top of the panel represent the individual plants for testing. The ${ }^{32} \mathrm{P}$-labeled RNA probe for the detection of BaMV RNA was complementary to the $3^{\prime}$ end of positive-strand RNA of BaMV. Data are mean BaMV gRNA level compared with the control shown below the panel. The ethidium-bromide-stained gel (bottom panel) shows ribosomal RNA (rRNA) as the loading control. C, Total protein was extracted from pKB-, pKB-RepHA-, or pKB-RepHA21infiltrated leaves at 4 days postagroinfiltration and separated by $12 \%$ sodium dodecyl sulfate polyacrylamide gel electrophoresis. Gels were stained with Coomassie blue (upper panel) or transferred to polyvinylidene difluoride membrane and probed with rabbit antiserum against BaMV TGBp1 (lower panel) $\mathrm{M}=$ prestained protein markers with molecular mass (in kilodaltons) shown on the left. Arrowheads indicate the positions of CP and TGBp1 in the upper and lower panel, respectively. RuBisCO large subunit (RbcL) is the loading control. 
plastid-targeted proteins (Lin et al. 2001). Both locate in the chloroplast stroma and are responsible for importing precursor

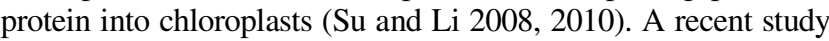
revealed that the transit peptides of AtcpHsp70-1 and AtcpHsp702 contain different age-selective signals that contribute to their preferential import into chloroplasts of different ages, which suggests that these proteins are also involved in age-dependent import of other associated proteins (Teng et al. 2012). These previous observations highlighted different important aspects of Hsps in the viral infection cycles and suggested the potential involvement of Hsps in age-related tissue preferences of plant viruses.

In this study, to further explore the possible contribution of Hsps to the replication and age-related tissue preferences of BaMV, we developed a system for transient overexpression of Rep as a bait to trap and analyze interacting Hsps in mature leaves of $N$. benthamiana. An isoform of $N$. benthamiana cpHsp70, NbcpHsp70-2, was found necessary for efficient BaMV replication in protoplasts. In addition, mislocalization of NbcpHsp70-2 reduced $\mathrm{BaMV}$ accumulation in plants, which suggests that NbcpHsp70-2 plays a role in associating BaMV replication complexes with chloroplasts. Furthermore, transient expression of an NbcpHsp70-2 mutant, with the transit peptide replaced with that for younger chloroplasts, enhanced BaMV accumulation in young leaves of $N$. benthamiana. These results suggest that NbcpHsp70-2 is required for efficient replication of BaMV in mature leaves and is involved in the virus's age-dependent tissue preference.

\section{RESULTS}

\section{Construction of recombinant BaMV infectious clones expressing HA-tagged Rep protein and identification of the critical octanucleotide region of TGB subgenomic RNA promoter.}

To isolate and characterize components of the replication complexes of BaMV, a coimmunoprecipitation strategy was adopted with an antibody specific to BaMV-Rep protein. However, the low accumulation of BaMV-Rep or its poor immunogenicity did not allow for direct serological detection even in the replicase activity-enriched fraction isolated from BaMV-infected plants. To circumvent the difficulty, a commonly used small tag, $\mathrm{HA}$, was fused to the C-terminal of Rep to facilitate the immunecapture of Rep-HA containing replication complexes with antiHA antibodies after infection of plants with the recombinant plasmid clone of BaMV, pKB-RepHA, by Agrobacteriummediated transient expression (agroinfiltration) (Fig. 1A). However, at 4 days postagroinfiltration (dpai), the BaMV gRNA accumulation in pKB-RepHA-infiltrated leaves was decreased to $32 \%$ of that in wild-type pKB-infiltrated ones (Fig. 1B). Of note, BaMV subgenomic RNA (sgRNA) for TGBp1 (TGBsgRNA) was not detectable in pKB-RepHA-infiltrated leaves; therefore, the HA fusion at the BaMV-Rep $\mathrm{C}$ terminus might have disrupted the TGBsgRNA transcription. On examining the upstream sequence of the BaMV TGB coding region (Fig. 1A), we found that the insertion of the HA coding sequence upstream of the stop codon of the Rep gene disrupted an octanucleotide element, GUUAAGUA, representing promoter-like sequences conserved among potexviruses for TGBsgRNA transcription (Kim and Hemenway 1997). To test whether the transcription of TGBsgRNA could be restored, pKB-RepHA21 was constructed with a 21-nucleotide fragment insertion downstream of the Rep gene to conserve the octanucleotide element (Fig. 1A). Inoculation assay revealed that, in pKB-RepHA21-infiltrated leaves, TGBsgRNA transcription was restored and gRNA accumulation was recovered to the wild-type level at 4 dpai (Fig. 1B). Furthermore, consistent with RNA accumulation, CP accumulation in pKB-RepHA21-infiltrated leaves was restored to the wild-type level, and the regenerated TGBsgRNA also could serve as the template for the translation of TGBp1 protein (Fig. 1C). Therefore, we have identified the position and verified the critical functions of the octanucleotide element in the BaMV TGBsgRNA promoter region and generated a recombinant BaMV construct, pKB-RepHA21, for producing HA-tagged Rep protein. For the following experiments, pKB-RepHA21 was used to infect $N$. benthamiana for analyzing host proteins in BaMV replication complexes by coimmunoprecipitation.

\section{Coimmunoprecipitation of NbcpHsp70 with BaMV-Rep-HA.}

To identify BaMV-Rep-HA-interacting host proteins in the membrane-associated replication complexes, BaMV RdRp preparations were isolated from pKB-RepHA21-infiltrated mature leaves of $N$. benthamiana at 4 dpai. The membranebound extracts (P30) underwent sucrose gradient fractionation, and three fractions with high RdRp activity were pooled and designated as F567. F567 was solubilized by using Sarkosyl (F567 $7_{\text {sark }}$ ) followed by ultracentrifugation to extract soluble proteins (S100). Both F567 sark and S100 underwent endogenous RdRp assays to examine RdRp activities. Most of the endogenous RdRp activities were extracted from P30 and retained in S100 after ultracentrifugation (Fig. 2A). Thus, the soluble fraction of S100 from BaMV RdRp preparation was chosen for coimmunoprecipitation experiments for its high RdRp activity. The S100 fractions prepared from the empty vector (EV, pKn)and pKB-RepHA21-infiltrated leaves were precleaned with Protein A-Sepharose to generate input samples (Fig. 2B). BaMVRep-HA could be specifically detected in the input prepared from pKB-RepHA21-infiltrated leaves but not EV-infiltrated ones, which suggests that the Rep-HA-containing complexes could be captured by using anti-HA antibody. Additionally, both S100 fractions from EV- and BaMV-RepHA21-infiltrated leaves contained host chaperones Hsp70, Hsp90, and cpHsp70, detected on immunoblotting assay with specific antibodies. After immunoprecipitation with anti-HA antibody, interactions between these chaperons and Rep-HA were detected only in the BaMVRepHA21 preparations (Fig. 2B), which suggests that these chaperones are associated specifically with Rep-HA in putative BaMV replication complexes. BaMV CP was also detected in the Rep-HA coimmunoprecipitated complexes, which is consistent with a previous finding (Lee et al. 2011). NbHsp90 was shown to interact with the BaMV RdRp domain on yeast two-hybrid analysis and glutathione $S$-transferase pull-down assay; the biofunction of $\mathrm{NbHsp90}$ in BaMV replication was also identified as positive regulator (Huang et al. 2012b). However, the copurification of $\mathrm{NbcpHsp70} \mathrm{in} \mathrm{the} \mathrm{replication} \mathrm{complexes} \mathrm{of} \mathrm{plant} \mathrm{viruses} \mathrm{has} \mathrm{not}$ been reported. Our results provide the first hint that NbcpHsp70 might also be involved in BaMV replication. Thus, we further investigated the role of NbcpHsp70 in BaMV replication and the stages for its involvement in the following experiments.

\section{Knocking down NbcpHsp70 caused a stunted phenotype} and reduced BaMV accumulation in $N$. benthamiana.

To demonstrate that the interaction between NbcpHsp70 and BaMV-Rep within the putative VRC is also relevant for BaMV replication in plants, we downregulated the expression of NbcpHsp70 by virus-induced gene silencing (VIGS). A 291-bp fragment from the coding sequence of NbcpHsp70 was cloned into a Tobacco rattle virus (TRV)-based silencing vector to generate TRV:cpHsp70, which was cointroduced into $N$. benthamiana with TRV1 via Agrobacterium-mediated infiltration. NbcpHsp70 expression was decreased to $37 \%$ of that of the mock-infiltrated plants (TRV:Luciferase [TRV:Luc]) at 10 dpai (Fig. 3A). However, downregulation of NbcpHsp70 
expression in $N$. benthamiana resulted in a dwarf phenotype (Fig. 3A), which indicates that NbcpHsp70 is essential for plant development. Next, to investigate the impact of NbcpHsp70 downregulation on BaMV accumulation in plants, systemic leaves from Luc- and NbcpHsp70-silenced $N$. benthamiana plants were agroinfiltrated with a BaMV infectious clone harboring green fluorescent protein (GFP), pKBG (Fig. 3B), and GFP signals were directly monitored at 2 dpai. Strong green fluorescence (GF) was observed from BaMV-GFP accumulation in Luc-silenced leaves. In contrast, GF in NbcpHsp70-silenced leaves was weak (Fig. 3B). To associate the observation of the GFP signals with the virus accumulation, the same infiltrated leaf discs were examined for accumulation of BaMV RNA by Northern blot analysis. The results are consistent with the intensity of GF (Fig. 3C); thus, knocking down NbcpHsp70 significantly reduced the accumulation of BaMV RNA.

\section{Specific silencing of NbcpHsp70-2 inhibits}

\section{BaMV replication in $N$. benthamiana protoplasts.}

Because the downregulation of NbcpHsp70 in N. benthamiana affected the development of the host, decreased accumulation of BaMV in NbcpHsp70-silenced plants may have been indirectly due to the effect on plant physiology. To exclude this possibility, we individually knocked down the expression of different NbcpHsp70 isoforms. In A. thaliana, knockout mutants of stromal Hsp70 cphsc70-1 and cphsc70-2 showed different phenotypes; however, the $c p h s c 70$ double knockout mutant was lethal, which suggests that these two stromal Hsp70 have distinct but also redundant functions ( $\mathrm{Su}$ and $\mathrm{Li}$ 2008). A search of the $N$. benthamiana transcriptome database (Nakasugi et al. 2013) for NbcpHsp70 genes revealed two putative cpHsp70 isoforms, NbcpHsp70-1 and NbcpHsp70-2. We first tested whether the expression of cpHsp70 isoforms was affected by the infection of
BaMV in $N$. benthamiana using real-time quantitative reversetranscription polymerase chain reaction (RT-PCR). The result revealed that the expression of both $\mathrm{cpHsp} 70$ isoforms increased during the course of BaMV infection. At the seventh day postBaMV infection (dpi), the expression of cpHsp70-1 and cpHsp702 significantly increased to eight- and threefold, respectively, that of mock inoculation (Supplementary Fig. S1). The nucleotide sequence identity between cpHsp70-1 and cpHsp70-2 coding sequences was $72 \%$ but only $5 \%$ between their $3^{\prime}$ UTR. Therefore, to design the specific silencing of corresponding isoforms, fragments of 3'UTR of NbcpHsp70-1 and NbcpHsp70-2 were individually cloned into the TRV-based VIGS vector. In this experiment, in addition to the Luc-silencing construct as a negative control, phytoene desaturase (PDS)-knockdown plants, with the photo-bleaching phenotype, were used as a positive control for VIGS (Fig. 4A and B).

To examine the specific RNA silencing of NbcpHsp70-1 and NbcpHsp70-2, we checked the gene expression of several NbHsp70 family members by semiquantitative RT-PCR with gene-specific primers (Supplementary Table S1) at 10 dpai. NbcpHsp70-1 silencing did not significantly affect the expression of any of these other NbHsp70 genes. Similarly, knocking down NbcpHsp70-2 did not affect the expression of the other $\mathrm{NbHsp} 70$ genes; therefore, the gene silencing was specific to NbcpHsp70-1 or NbcpHsp70-2 when infiltrated with the corresponding clones (Fig. 4A). Notably, silencing NbcpHsp70-1 or NbcpHsp70-2 did not result in a stunted phenotype. However, NbcpHsp70-2 silencing caused more severe yellowing on upper leaves compared with NbcpHsp70-1 silencing (Fig. 4B). The yellowing phenotype might be caused by the reduced chlorophyll content.

To determine which cpHsp70 isoform would affect BaMV replication, BaMV RNA was inoculated into protoplasts derived

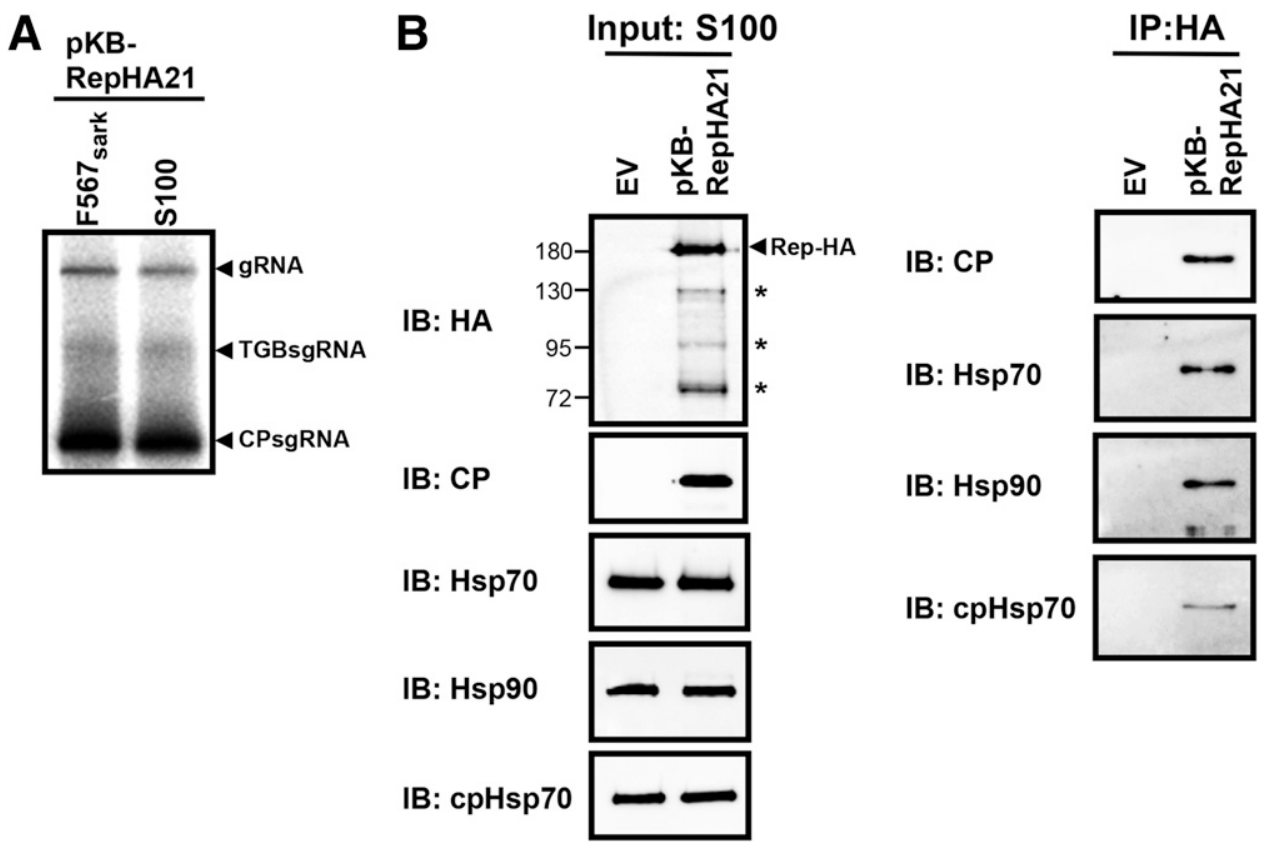

Fig. 2. Coimmunoprecipitation of various cellular chaperones with Bamboo mosaic virus (BaMV) replication protein (Rep)-HA. A, Activities of BaMV RNA-

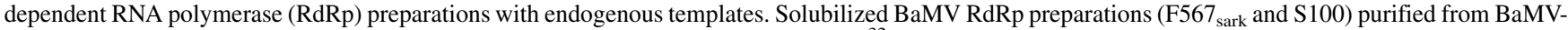
RepHA21-infected leaves underwent RdRp activity assay with endogenous templates. ${ }^{32} \mathrm{P}$-labeled reaction products were electrophoresed on $1 \%$ agarose gel and analyzed by using PhosphorImager. Arrowheads indicate the positions of synthesized products from endogenous templates: genomic RNA (gRNA), triple gene block subgenomic RNA (TGBsgRNA), and capsid protein (CP)sgRNA, respectively. B, Immunoprecipitation of the Rep-HA containing complexes with anti-HA antibody. Input = the S100 prepared from empty vector (EV, pKn)- and pKB-RepHA21-infiltrated leaves after precleaning with Protein A-Sepharose. Equal amounts of input proteins were used in immunoblotting (IB) with antibodies against HA, BaMV CP, Hsp70, Hsp90, and cpHsp70. Sizes of protein markers are indicated at the left. The arrowhead shows the position of Rep-HA. Presumed degraded forms of Rep-HA are indicated by asterisks (*). After immunoprecipitation (IP) with anti-HA antibody, the presence of BaMV CP, Hsp70, Hsp90, and cpHsp70 in coimmunoprecipitates was detected with corresponding antibodies by immunoblotting. 
from control (TRV:Luc or TRV:PDS) or NbcpHsp70-knockdown plants. Total RNA was extracted from protoplasts at $24 \mathrm{~h}$ postinoculation for Northern blot analysis. We found 78 and $25 \%$ decreased accumulation of BaMV RNA in NbcpHsp70-1 and NbcpHsp70-2 knockdown protoplasts, respectively, compared with Luc-silenced protoplasts (Fig. 4C). Therefore, BaMV RNA accumulation was significantly reduced by the silencing of NbcpHsp70-2 but not NbcpHsp70-1 (Fig. 4C). These observations demonstrate that specific downregulation of NbcpHsp70-2 would inhibit BaMV replication in $N$. benthamiana protoplasts. Moreover, BaMV accumulation in protoplasts derived from PDS-silenced plants did not significantly differ from that in mock-silenced protoplasts (Fig. 4C), which suggests that the inhibition of BaMV replication in NbcpHsp70-2 knockdown protoplasts was not due to the chlorophyll reduction effects.

\section{Transient expression of the mislocalized NbcpHsp70-2 reduces BaMV accumulation in $N$. benthamiana.}

To characterize the possible roles of the NbcpHsp70-2 involved in various stages of BaMV replication, we cloned the full-length NbcpHsp70-1 and NbcpHsp70-2 genes. Amino acid sequence alignment showed $83 \%$ identity between NbcpHsp70-1 and NbcpHsp70-2 but the N-terminal transit peptide sequences were highly variable (Supplementary Fig. S2). Because of significant inhibition of BaMV accumulation only in NbcpHsp70-2 knockdown protoplasts, the possible role of NbcpHsp70-2 in $\mathrm{BaMV}$ replication might be related to the function of the transit peptide (predicted to be 82 amino acids at the $\mathrm{N}$ terminus) involved in import of NbcpHsp70-2 into chloroplasts. To test the hypothesis, we fused the wild-type NbcpHsp70-2 and a transit peptide deletion mutant $(\triangle \mathrm{TPcpHsp} 70-2)$ with the orange fluorescent protein (OFP) on the $\mathrm{C}$ terminus for transient expression of fusion proteins cpHsp70-2-OFP and $\Delta$ TPcpHsp70-2-OFP. On confocal microscopy of the subcellular localization of these fusion proteins in $N$. benthamiana protoplasts, orange fluorescent signals were observed in chloroplast stroma in protoplasts transiently expressing cpHsp70-2-OFP; however, $\triangle$ TPcpHsp70-2OFP was mislocalized in cytoplasm, which demonstrates the function of the transit peptide for import of cpHsp70-2 to chloroplasts (Fig. 5A).
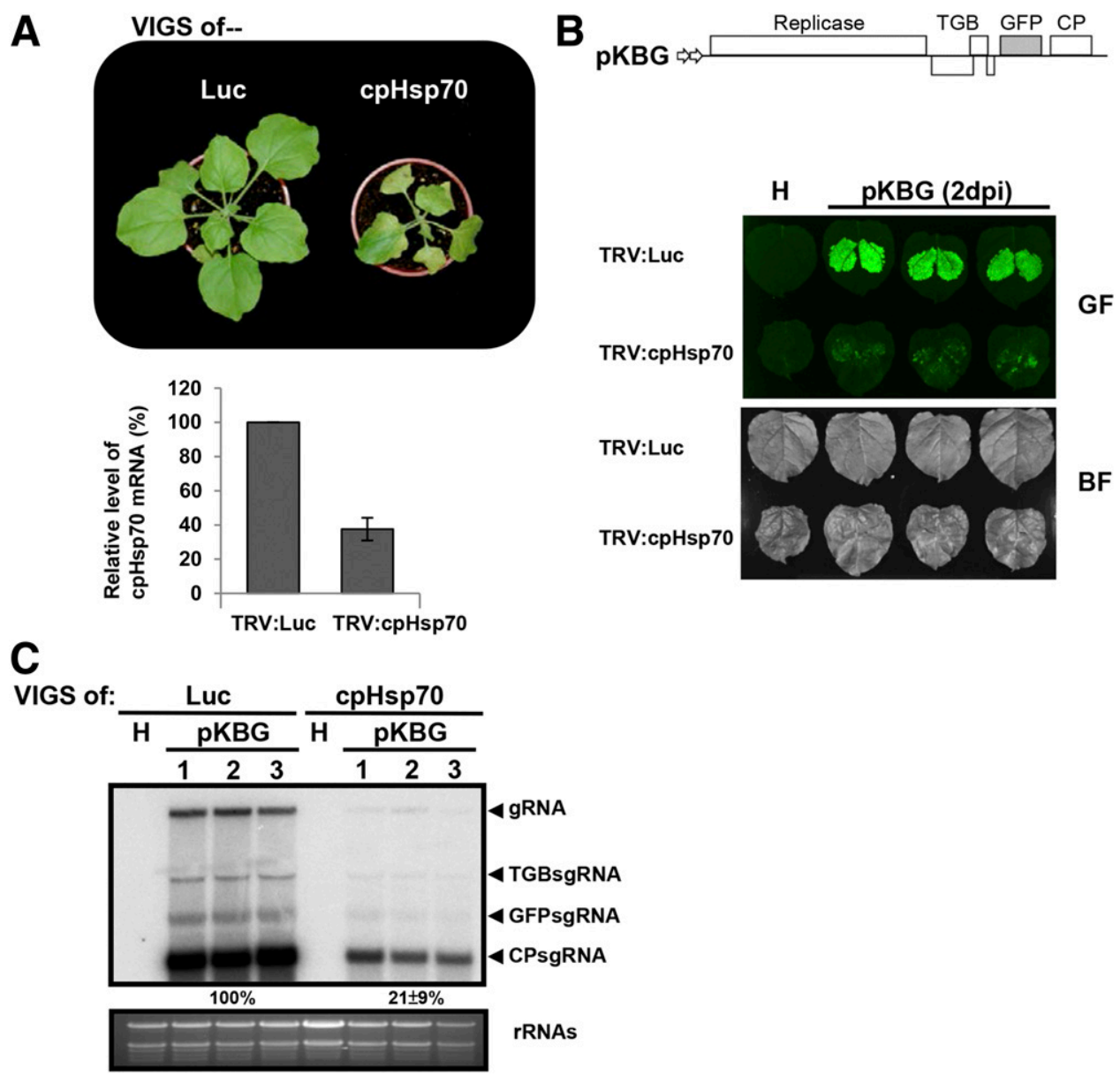

Fig. 3. Effect of cpHsp70-knockdown on Bamboo mosaic virus (BaMV) accumulation. A, Phenotype of cpHsp70-knockdown Nicotiana benthamiana. The Tobacco rattle virus (TRV)-based virus-induced gene silencing (VIGS) system was used to silence Luciferase (Luc) and cpHsp70. As compared with Luc-knockdown plants (control), cpHsp70-knockdown plants showed a dwarf phenotype (upper panel). Real-time polymerase chain reaction of relative accumulation of NbcpHsp70 mRNA in control (TRV:Luc) or cpHsp70-knockdown (TRV:cpHsp70) plants at 10 days postagroinfiltration (dpai). Data are mean \pm standard deviation of three independent assays. B, Green fluorescent protein (GFP) fluorescence expression in cpHsp70-knockdown leaves. Upper panel shows the genome organization of pKBG. The subgenomic RNA (sgRNA) of GFP is driven by the promoter of capsid protein (CP)sgRNA. Agrobacterium tumefaciens strain GV3850 carrying pKBG was infiltrated into VIGS-Luc (TRV:Luc) and VIGS-cpHsp70 (TRV:cpHsp70) leaves (the third leaf above infiltrated leaves) at 10 dpai. GFP fluorescence (GF) and bright-field (BF) images were taken by using the Fujifilm luminescent image analyzer LAS4000 on silenced and control plants at 2 dpai of pKBG. TGB $=$ triple gene block. C, Northern blot analyses of the accumulation of BaMV-GFP in VIGSmediated cpHsp70-knockdown plants. At 10 dpai, the upper leaves of control (TRV:Luc) or silenced (TRV:cpHsp70) plants were infiltrated with pKBG. The infiltrated leaves were tested at 2 dpai for BaMV accumulation by Northern blot analysis. The ${ }^{32}$ P-labeled RNA probe for detecting BaMV RNA was complementary to the $3^{\prime}$ end of positive-strand RNA of BaMV. Data are mean BaMV genomic RNA (gRNA) accumulation compared with the control shown below the panel. The ethidium-bromide-stained gel before blotting shows ribosomal RNA (rRNA) as the loading control. 
To understand the effect of cpHsp70-2 subcellular localization on BaMV infection, cpHsp70-2 and $\triangle \mathrm{TPcpHsp70-2}$ were overexpressed in $N$. benthamiana. After transient expression for 1 day, the expression of the control protein (GFP-His), cpHsp702-His, and $\triangle \mathrm{TPcpHsp70-2-His} \mathrm{was} \mathrm{verified} \mathrm{by} \mathrm{immunoblotting}$ (Fig. 5B and C). The infectious clone of BaMV, pKB, was then infiltrated into these leaves. At 2 dpai, BaMV RNA was examined by Northern blot analysis. Overexpression of cpHsp70-2 did not aid BaMV infection (Fig. 5D), which suggests that the amount of endogenous cpHsp70-2 might already be sufficient to support BaMV replication. However, BaMV accumulation was lower in plants transiently expressing $\Delta \mathrm{TPcpHsp} 70-2$ than in GFP-expressing plants (Fig. 5E). Thus, the proper localization of cpHsp70-2 into chloroplasts is required for efficient accumulation of BaMV in $N$. benthamiana.
BaMV-Rep interacts with NbcpHsp70-2.

The above results revealed the importance of chloroplast importation of NbcpHsp70-2 for efficient BaMV accumulation. Transient expression of $\Delta$ TPcpHsp70-2 has a dominant-negative effect on BaMV accumulation, which suggests that this mislocalized cpHsp70-2 might sequester BaMV-Rep or factors required for BaMV replication in cytoplasm. To test this hypothesis, we used a GFP pull-down assay with transiently expressed BaMV-Rep to determine whether BaMV-Rep can interact with cpHsp70-2 or $\triangle \mathrm{TPcpHsp70-2}$ in the absence of BaMV RNA to exclude the confounding effects. GFP was fused to BaMV-Rep at the $\mathrm{C}$ terminus for transient coexpression of Rep-GFP with cpHsp70-2-OFP or $\triangle \mathrm{TPcpHsp70-2-OFP}$ for 2 days. Total protein extracts (input) were subjected to GFP-binding protein-coupled magnetic agarose beads, followed by washing and elution of the
A

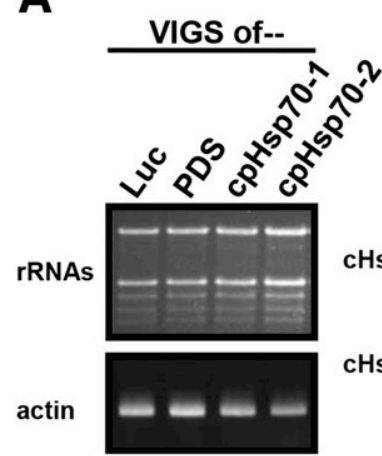

cHsp70-3

cHsp70-4

cHsp70-5

cHsp70-6
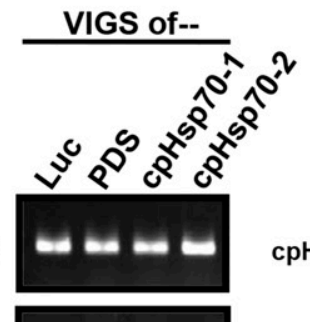

-
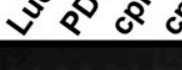

.

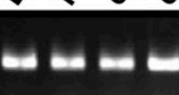

cpHsp70-1

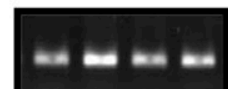

cpHsp70-2

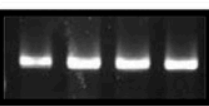

erHsp70-1

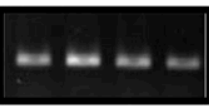

erHsp70

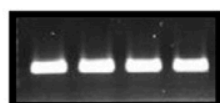

erfor 70

$\mathrm{mtHsp} 70-1$

VIGS of--

B

VIGS of--

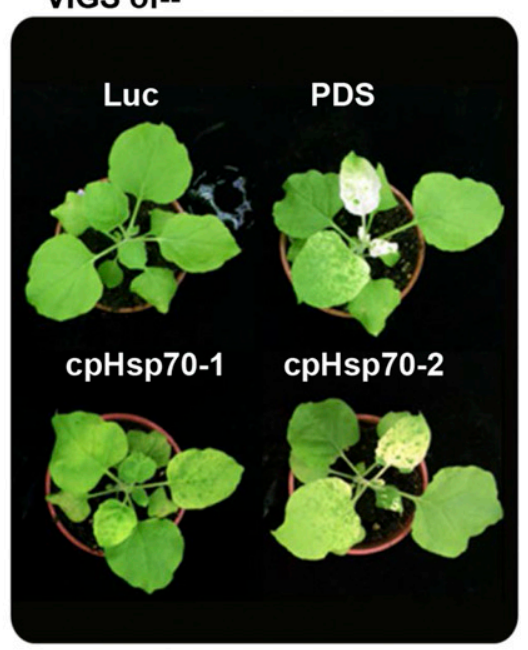

\section{VIGS of--}

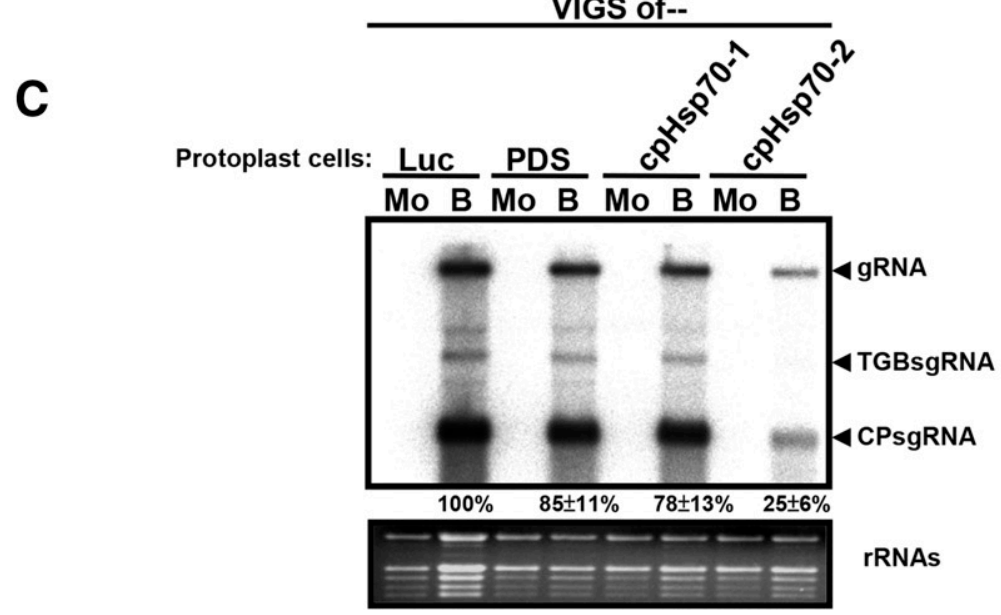

Fig. 4. Effect of NbcpHsp70-1 and NbcpHsp70-2 knockdown on Bamboo mosaic virus (BaMV) accumulation in Nicotiana benthamiana protoplasts. A, Semiquantitative reverse-transcription polymerase chain reaction analysis of mRNA levels of Hsp70 gene family in silenced (cpHsp70-1 and cpHsp70-2) and control (Luciferase [Luc] and phytoene desaturase [PDS]) plants. Actin was an internal control. B, Phenotypes were observed in NbcpHsp70-1- and NbcpHsp70-2-knockdown $N$. benthamiana. No visible phenotype was observed in the cpHsp70-1 knockdown plants; in contrast, the upper leaves of cpHsp702-knockdown plants showed yellowing. C, Northern blot analysis of the BaMV RNA accumulation in virus-induced gene silencing (VIGS)-mediated $N$. benthamiana protoplasts with gene knockdown. Protoplasts from Luc-, PDS-, cpHsp70-1, or cpHsp70-2 knockdown plants were inoculated with $1 \mu \mathrm{g}$ of viral RNA of BaMV. The RNA samples of protoplasts were analyzed at $24 \mathrm{~h}$ postinoculation. Data are mean BaMV genomic RNA (gRNA) accumulation compared with the control shown below the panel. TGB = triple gene block, sgRNA = subgenomic RNA, CP = capsid protein, and rRNA = ribosomal RNA. 


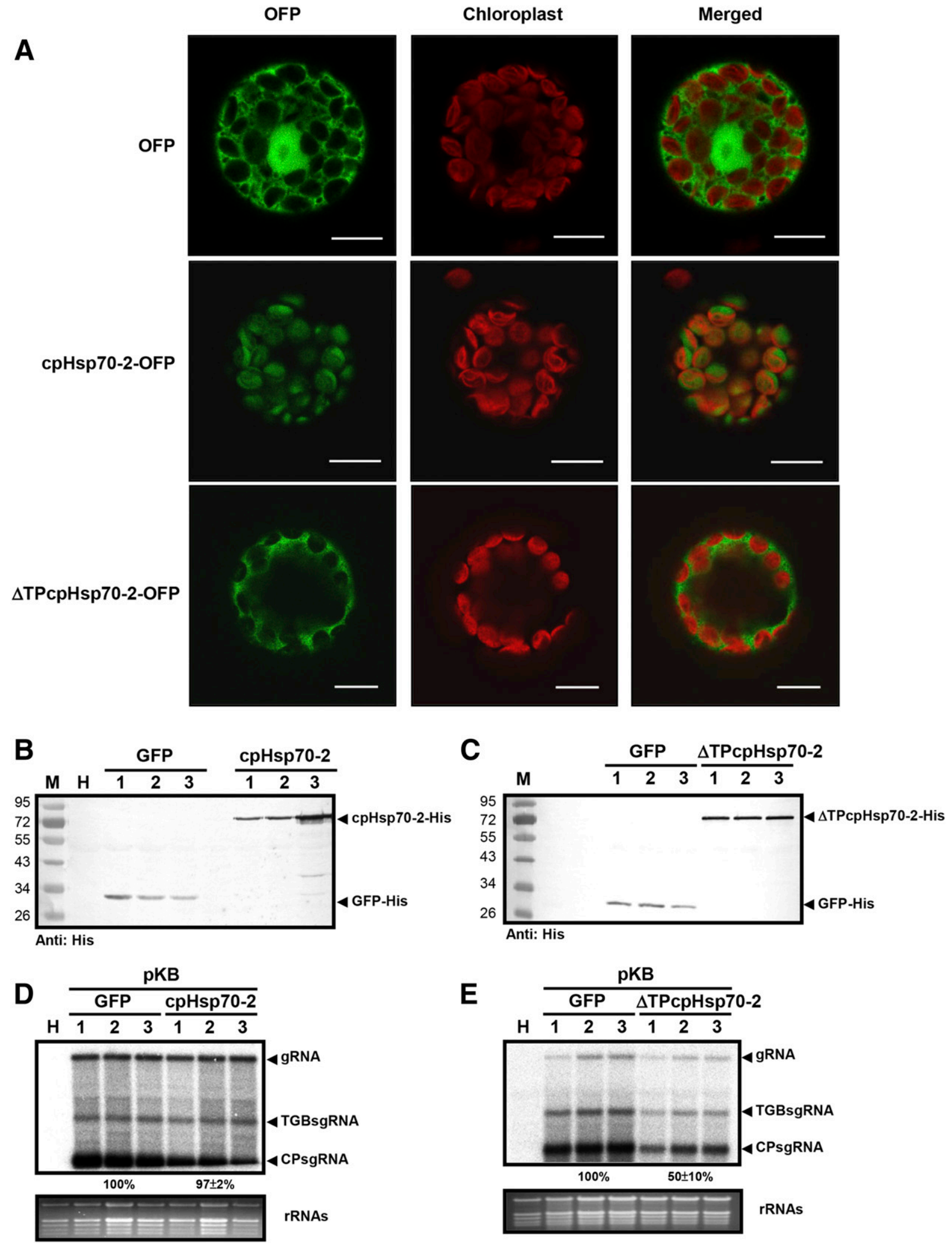

Fig. 5. Effect of transiently expressed wild-type and mislocalized NbcpHsp70-2 on Bamboo mosaic virus (BaMV) accumulation. A, Subcellular localization of cpHsp70-2 and $\Delta \Delta$ TPcpHsp70-2 in Nicotiana benthamiana protoplasts. Orange fluorescent protein (OFP), cpHsp70-2 fused with OFP (cpHsp70-2-OFP), and $\Delta$ TPcpHsp70-2-OFP were transiently expressed in $N$. benthamiana. Protoplasts were prepared at 3 days postagroinfiltration (dpai) and examined by confocal microscopy. Bar $=10 \mu \mathrm{m}$. B and C, Immunoblot analyses of transiently expressed control protein (green fluorescent protein [GFP]-His), cpHsp70-2-His, and the mutant without transit peptide ( $\triangle \mathrm{TP} \mathrm{PpHsp} 70-2-\mathrm{His})$ at 1 dpai by Agrobacterium-mediated expression. Total proteins were separated on $12 \%$ sodium dodecyl sulfate polyacrylamide gel electrophoresis and monoclonal anti-His antibody was used to detect transiently expressed proteins. D and E, BaMV RNA accumulations in plants transiently expressing wild-type and mislocalized cpHsp70-2, respectively. After transient expression of GFP-His, cpHsp70-2-His, and $\Delta \mathrm{TPcpHsp70-2-His} \mathrm{for} 1$ day, infiltrated leaves were infiltrated with pKB. Total RNA was isolated at day 2 after BaMV infection, and BaMV RNA accumulation was detected by Northern blot analysis. Data are mean BaMV genomic RNA (gRNA) accumulation compared with the control shown below the panel. $\mathrm{TGB}=$ triple gene block, sgRNA $=$ subgenomic RNA, $\mathrm{CP}=$ capsid protein, and $\mathrm{rRNA}=$ ribosomal RNA . 
GFP pull-down complexes. Immunoblotting revealed that both cpHsp70-2-OFP and $\triangle$ TPcpHsp70-2-OFP could be detected by anti-OFP antibody in the GPF pull-down fractions (Fig. 6), indicating that the mature form of cpHsp70-2, without the transit peptide, may still interact with BaMV-Rep-GFP. This result suggested that the overexpression of $\Delta \mathrm{TPcpHsp70-2}$ could sequester BaMV-Rep in cytoplasm, which would interfere with the transportation of BaMV-Rep to the appropriate replication site and, therefore, inhibit BaMV accumulation.

\section{Overexpression of the transit peptide mutant of NbcpHsp70-2 downregulated BaMV RNA accumulation in older leaves of $N$. benthamiana.}

The above results demonstrated that BaMV could selectively exploit NbcpHsp70-2 but not NbcpHsp70-1 for replication. In A. thaliana, AtcpHsp70-1 is imported into chloroplasts of older leaves preferentially, which is regulated by two consecutive positively charged amino acids (KR) of its transit peptide. Mutation of KR in AtcpHsp70-1 abolished the preference for the importation into chloroplasts of older leaves (Teng et al. 2012). The transit peptide sequence of NbcpHsp70-2, similar to that of AtcpHsp70-1, also contains two consecutive positively charged residues, Arg24 and Lys25, designated RK (Fig. 7A). During our studies of BaMV replication, we observed that BaMV exhibits an age-dependent tissue preference, accumulating to a higher level in older than in younger leaves (Fig. 8A), which is contrary to observations for many other plant viruses such as PVX and PVY that accumulate to higher levels in younger leaves (Venekamp and Beemster 1980; Venekamp et al. 1980).

To determine whether the age-dependent preference of BaMV accumulation in older leaves could be regulated by the

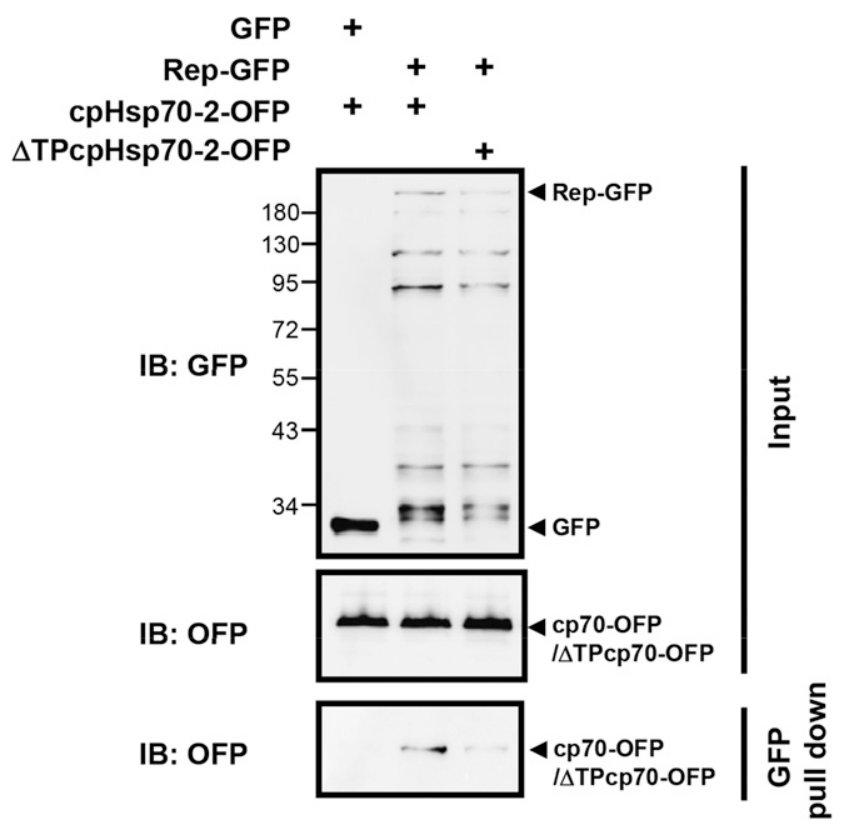

Fig. 6. Interaction between Bamboo mosaic virus (BaMV) replication protein (Rep) and NbcpHsp70-2. Transiently expressed proteins green fluorescent protein (GFP), replication protein (Rep)-GFP, cpHsp70-2 orange fluorescent protein (OFP), and $\triangle \mathrm{TPcpHsp70-2-OFP}$ were coexpressed with the combinations indicated above each lane. At 2 days postagroinfiltration (dpai), total protein (input) was isolated for GFP pull-down assays. Input proteins were detected by immunoblotting with antibodies against GFP and OFP. After incubation with GFP-Trap and washing, the GFP binding protein-bound complexes were eluted by boiling, separated on $10 \%$ sodium dodecyl sulfate polyacrylamide gel electrophoresis, and analyzed by immunoblotting (IB) with antibodies specific to OFP (bottom panel). transit peptide sequence of NbcpHsp70-2, we replaced the two positively charged RK in the cpHsp70-2 transit peptide with Glu, designated as RK2425EE, which should reduce the importation efficiency of NbcpHsp70-2 into older chloroplasts. OFP was fused to the C terminus of RK2425EE as a reporter for observing the subcellular localization of the transiently expressed fusion proteins in older leaves of $N$. benthamiana by confocal microscopy. The OFP signals clearly indicated that wild-type cpHsp70-2 was localized in chloroplasts and $\Delta \mathrm{TPcpHsp} 70-2$ was in cytoplasm (Fig. 7B). However, orange fluorescence of RK2425EE-OFP was observed in both chloroplasts and cytoplasm (Fig. 7B), which suggests that the transit peptide mutation decreased the efficiency of cpHsp70-2 transportation into older chloroplasts. Leaves transiently expressing RK2425EE showed the mature or precursor form of cpHsp70-2 accumulation; therefore, only a portion of RK2425EE was imported into chloroplasts and processed into the mature form (Fig. 7C).

Next, we infiltrated pKB into $N$. benthamiana transiently expressing GFP-His, $\Delta$ TPcpHsp70-2-His, or RK2425EE-His and found decreased accumulation of BaMV RNA in plants transiently expressing $\triangle \mathrm{TPcpHsp} 70-2$ or RK2425EE to 37 and $41 \%$, respectively, of that in GFP-expressing plants (Fig. 7D). Therefore, consistent with the effects of $\triangle T$ TPcpHsp70-2 on BaMV accumulation in $N$. benthamiana, the altered age-dependent import regulation signal decreased the efficiency of cpHsp70-2 import into older chloroplasts which, in turn, downregulated BaMV accumulation in older leaves of $N$. benthamiana.

\section{Transient expression of an NbcpHsp70-2 mutant with transit peptide replaced by that for transportation into younger chloroplasts positively regulated BaMV accumulation in younger leaves of $N$. benthamiana.}

The alteration of key amino acids in the cpHsp70-2 transit peptide interfered with the importation into older chloroplasts and reduced BaMV RNA accumulation, which implies that cpHsp70-2 might contribute to the age-dependent preference of BaMV accumulation in older leaves of $N$. benthamiana. To confirm whether the older leaves are more susceptible to BaMV infection, we infiltrated pKB-RepHA in leaves at different ages: the third (L3), fifth (L5), and sixth leaves (L6) from the bottom to top along the stem of seedlings with decreasing degrees of maturity (Fig. 8A). The movement-deficient BaMV infectious clone pKB-RepHA (Fig. 1) was used as the inoculum to prevent the cell-to-cell and systemic movement of BaMV into neighboring cells or leaves, so that the accumulation levels of BaMV in each inoculated leaf would not be confounded by that moving in from neighboring leaves. At 3 and 6 dpai, total RNA was extracted from infiltrated leaves and subjected to Northern blot analyses for detecting BaMV RNA accumulation. BaMV infection was more efficient in L3 than L5 and L6. The difference was most obvious in L6, with BaMV RNA accumulation decreased to 22.4 and $16.8 \%$ of that in L3 at 3 and 6 dpai, respectively (Fig. 8A). Therefore, mature leaves were more susceptible than younger leaves to BaMV replication.

To verify the association between the preference for importation of cpHsp70-2 into older chloroplasts and the preferential accumulation of BaMV in older leaves, we examined the interactions between BaMV-Rep complexes and cpHsp70-2 in different leaf positions. L3 and L6 were individually infected with BaMV-RepHA21 and coinfiltrated with cpHsp70-2-OFP. At 2 dpai, BaMV-Rep-HA-associated replication complexes were purified and precipitated with anti-HA antibody to test the association of $\mathrm{cpHsp70-2.} \mathrm{Western} \mathrm{blot} \mathrm{analysis} \mathrm{was} \mathrm{performed}$ with antibodies specific to HA tag or OFP to ensure the accumulation levels of Rep-HA or cpHsp70-2 in the S100 input fractions (Fig. 8B, left panel). After immunoprecipitation with anti-HA antibody, approximately equal amounts of Rep-HA 
A

transit peptide seq.

AtcpHsp70-1: MASSAAQIHVLGGIGFASSSSSKRNLNGKGGTFMPRSAFFGTRTGPFSTPTSAFLRMGTRNGGGASRYAVGPVRVVN

NbcpHsp70-2: MASSTAQIHALGATYFTTSSSSSRKPSKTVFLGQNINNRTLAFGLKQKKSSFSRRNGGGHAPLRVVAEKVVGIDLGTTNSAV

B

OFP
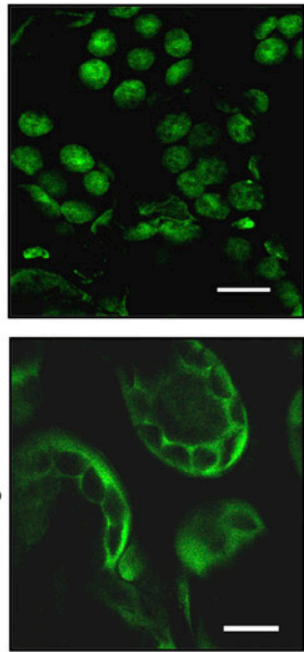

$\Delta$ TPcpHsp70-2-OFP

K2425EE-OFP
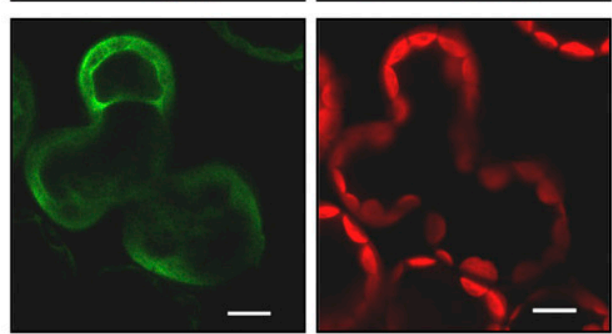

Chloroplast
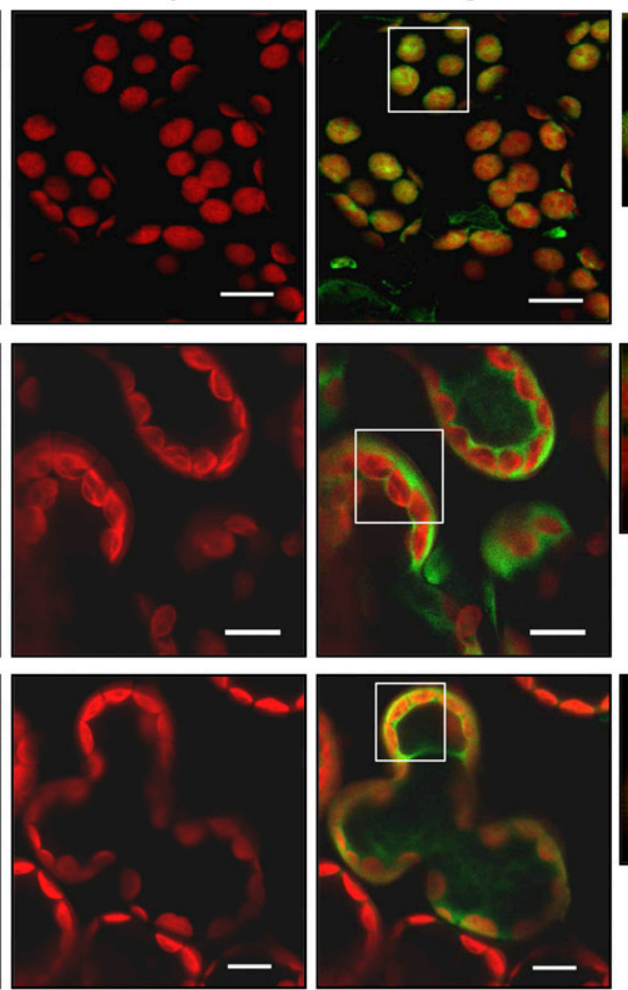

Zoom
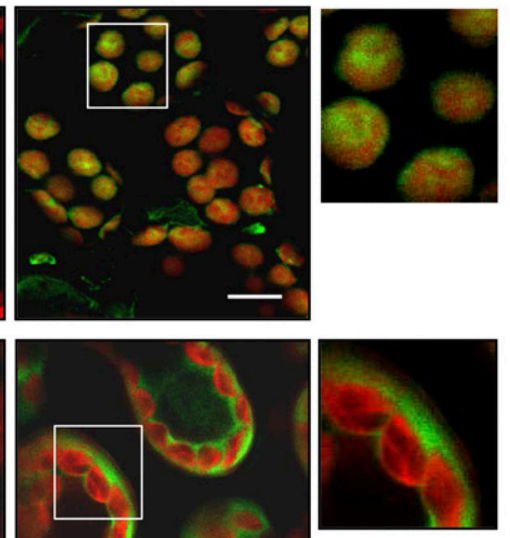

C

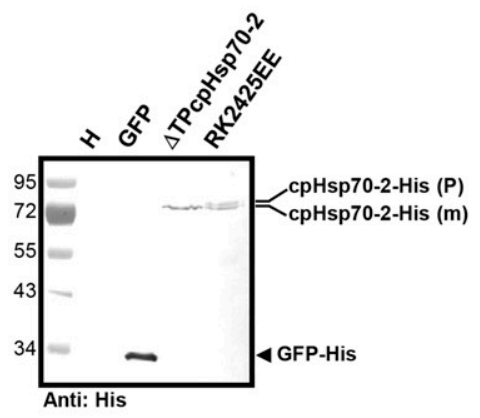

D

pKB
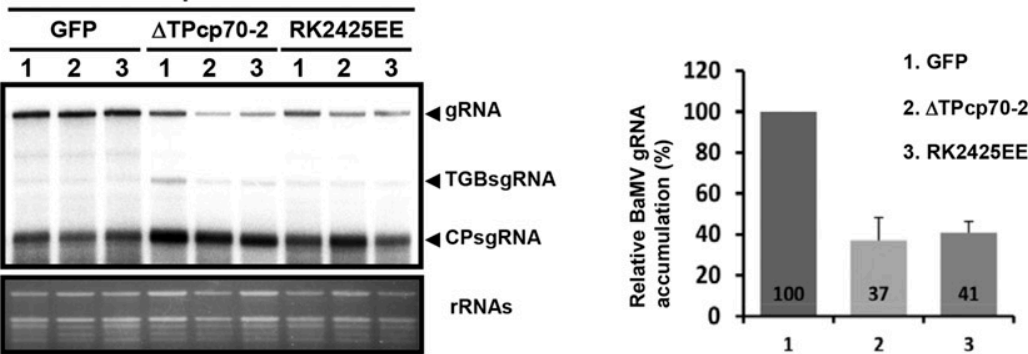

Fig. 7. Effect of transiently expressed NbcpHsp70-2 derivatives on Bamboo mosaic virus (BaMV) accumulation in Nicotiana benthamiana. A, Transit peptide sequences of Arabidopsis thaliana cpHsc70-1 (AtcpHsp70-1) and NbcpHsp70-2. Two consecutive positive-charge amino acids, underlined, determine the preferential import of AtcpHsp70-1 into older chloroplasts. B, Subcellular localization of cpHsp70-2, $\Delta$ TPcpHsp70-2, and RK2425EE in N. benthamiana leaves. Confocal microscopy of orange fluorescent protein (OFP) signals in $N$. benthamiana leaves transiently expressing cpHsp70-2-OFP, $\Delta$ TPcpHsp70-2OFP, or RK2425EE-OFP at 3 days postagroinfiltration (dpai). The white box indicates the merged region magnified, as shown in the panel labeled "zoom". $\mathrm{Bar}=10 \mu \mathrm{m}$. C, Immunoblotting of transiently expressed green fluorescent protein (GFP)-His, $\Delta \mathrm{TPcpHsp} 70-2-\mathrm{His}$, and transit peptide substitution mutant of cpHsp70-2 (RK2425EE-His) at 1 dpai by Agrobacterium-mediated expression. D, Northern blot analysis of BaMV RNA accumulation in N. benthamiana transiently expressing GFP-His, $\Delta$ TPcpHsp70-2-His, and RK2425EE-His. Ethidium-bromide-stained ribosomal RNA (rRNA) was a loading control. Numbers at the base of each bar are mean relative BaMV genomic RNA (gRNA) accumulation from three independent experiments (right panel). TGB $=$ triple gene block, sgRNA = subgenomic RNA, and $\mathrm{CP}=$ capsid protein. 
pull-down complexes were used to detect cpHsp70-2 (Fig. 8B, right panel, IB: HA). Less cpHsp70-2 was detected in the L6 sample than in the L3 sample (Fig. 8B, right panel, IB: OFP), which suggests that the association of cpHsp70-2 with BaMVRep complexes was decreased in younger leaves.
To further corroborate the hypothesis, we constructed a cpHsp70-2 mutant with its transit peptide replaced with that of RuBisCO small subunit (RbcS), which is preferentially imported into younger chloroplasts (Teng et al. 2012). We then infiltrated pKB into younger leaves (L6) that transiently expressed control

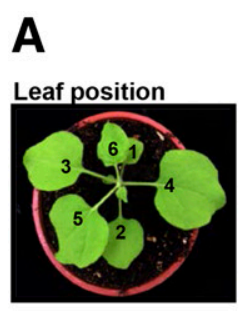

B
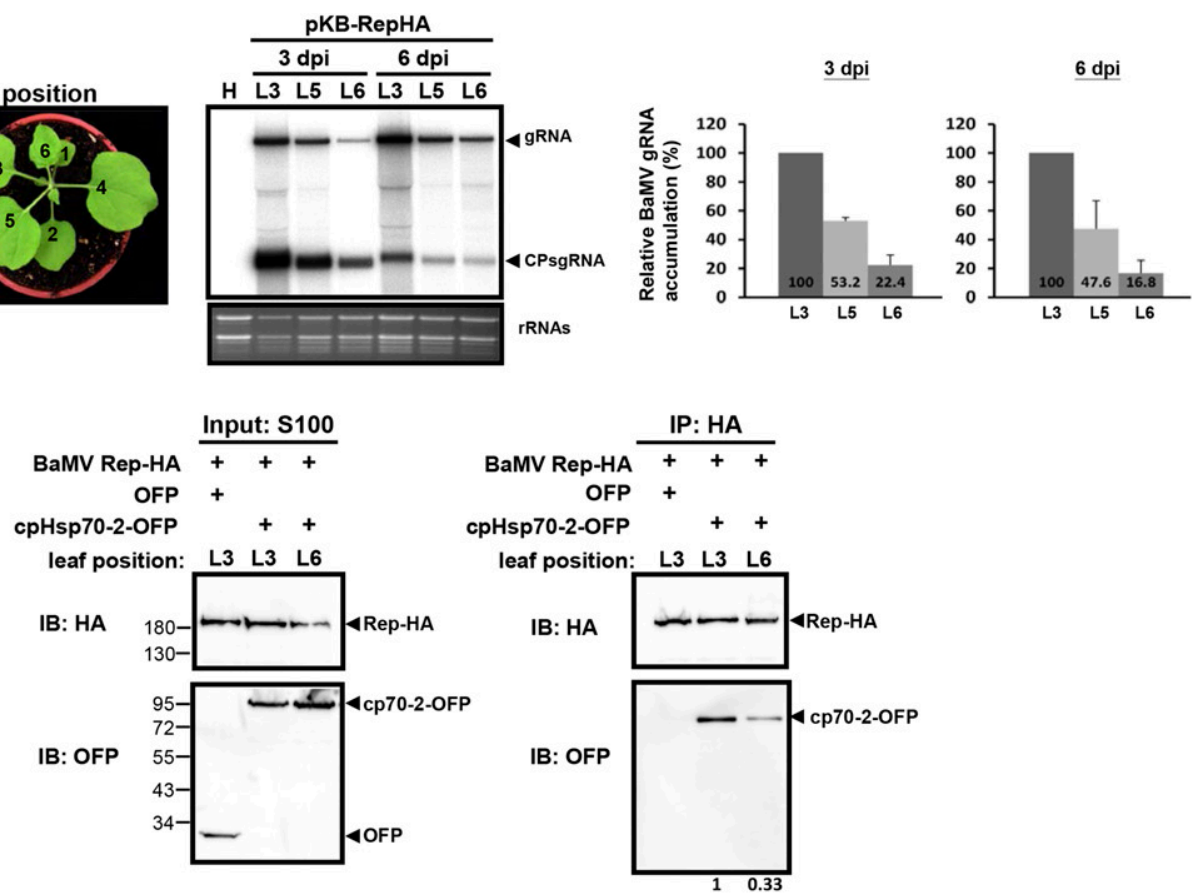

C
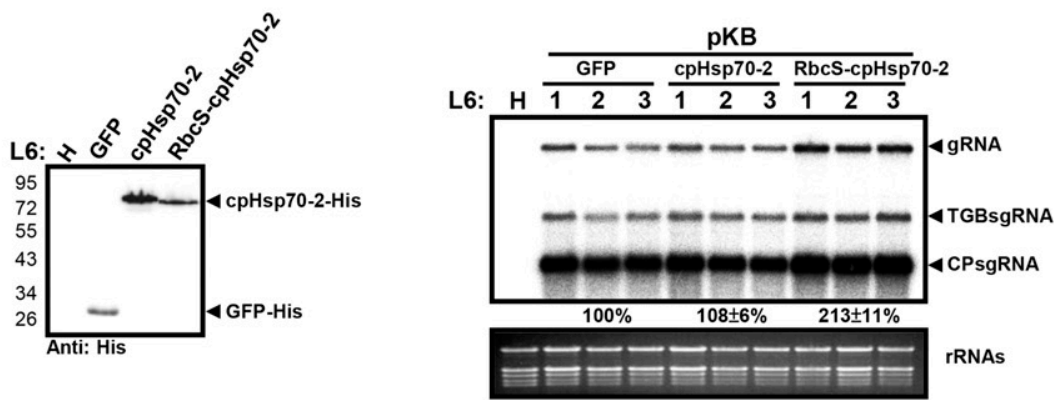

D
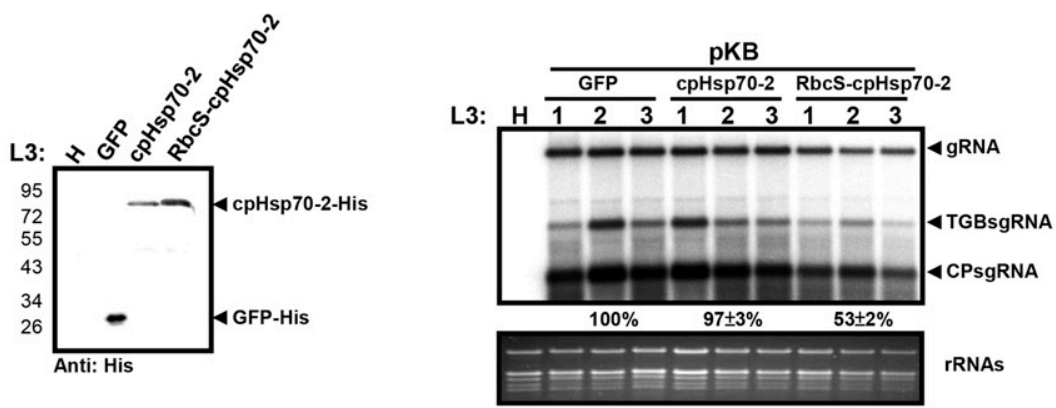

Fig. 8. Effect of transiently expressed NbcpHsp70-2 derivatives on Bamboo mosaic virus (BaMV) accumulation in young leaves of Nicotiana benthamiana. A, RNA accumulation of BaMV-replication protein (Rep)HA in Nicotiana benthamiana leaves of different positions. Different positions of $N$. benthamiana leaves (L3, L5, and L6), numbered on each leaf (left panel), were infiltrated with pKB-RepHA, a movement-deficient mutant of BaMV. Total RNA was isolated at 3 and 6 days postagroinfiltration (dpai) for Northern blot analysis with probes complementary to BaMV $3^{\prime}$ untranslated region (UTR) (middle panel). Numbers at the base of each bar are the mean relative level of BaMV genomic RNA (gRNA) accumulation from three independent experiments (right panel). B, Coimmunoprecipitation assay to verify the interaction between BaMV-Rep and cpHsp70-2 under BaMV infection conditions in leaves of different ages. Input $=$ the S100 prepared from leaves that were infected with BaMV-RepHA21 and coinfiltrated with constructs expressing orange fluorescent protein (OFP) or cpHsp70-2-OFP. Equal amounts of input proteins were used in immunoblotting (IB) assay with antibodies against HA and OFP. After immunoprecipitation (IP) with anti-HA antibody, equal amounts of Rep-HA precipitation underwent IB assays with corresponding antibodies. C and D, Immunoblot analysis of transiently expressed green fluorescent protein (GFP)-His, cpHsp70-2-His, or RbcS-cpHsp70-2-His in L6 (C, left panel) or L3 (D, left panel) at 1 dpai by Agrobacterium-mediated expression. Total proteins were separated by $12 \%$ sodium dodecyl sulfate polyacrylamide gel electrophoresis, transferred to polyvinylidene difluoride membrane, and probed with anti-His antibody. Northern blot analysis of BaMV RNA accumulation in L6 (C, right panel) or L3 (D, right panel) transiently expressing GFP-His, cpHsp70-2-His, or RbcS-cpHsp70-2-His. Ethidium-bromide-stained ribosomal RNA (rRNA) was a loading control. Data are mean BaMV gRNA accumulation compared with the control shown below the panel. TGB = triple gene block, sgRNA = subgenomic RNA, and CP = capsid protein. 
protein (GFP-His), cpHsp70-2-His, or RbcS-cpHsp70-2-His (Fig. 8C, left panel). BaMV RNA accumulation was increased to approximately $213 \%$ of that in plants expressing GFP control protein, which suggests that the transit peptide of $\mathrm{RbcS}$ directed the import of cpHsp70-2 to younger chloroplasts and facilitated BaMV accumulation in younger leaves (Fig. 8C, right panel). In contrast, BaMV RNA accumulation in older leaves (L3) expressing RbcS-cpHsp70-2-His was decreased (Fig. 8D, right panel). This result implied that, in older leaves, the transit peptide of RbcS might decrease the transportation efficiency of cpHsp70-2 into chloroplasts and thereby inhibit BaMV accumulation in older leaves.

To further confirm this hypothesis, the subcellular localization of cpHsp70-2 and RbcS-cpHsp70-2 were examined in younger and older leaves, respectively. Confocal microscopy was used to observe the OFP signals from cpHsp70-2-OFP or RbcScpHsp70-2-OFP transiently expressed at 3 dpai. NbcpHsp70-2 was preferentially imported into chloroplasts of older leaves (L6) whereas RbcS-cpHsp70-2 was preferentially imported into younger ones (L3) (Supplementary Fig. S3). These data were consistent with the above observations that showed that agedependent import of $\mathrm{cpHsp70-2}$ is regulated by the signal peptide of cpHsp70-2. Thus, the signal peptide of cpHsp70-2 might contribute to the age-dependent preference of BaMV accumulation in older leaves of $N$. benthamiana.

\section{DISCUSSION}

Age-dependent resistance or susceptibility to virus infections has been an important topic in animal viruses (Griffin 2016; Hirsch et al. 1970; Labrada et al. 2002), found largely related to the immune systems of animal hosts at different ages. In contrast, with plant viruses, much less attention has been paid to this topic, except for a few earlier reports (Venekamp and Beemster 1980; Venekamp et al. 1980), possibly because of the lack of proper systems or approaches to study the underlying mechanism. During our studies on BaMV, we noticed that, unlike other plant viruses that accumulate to high levels in younger leaves (Venekamp and Beemster 1980; Venekamp et al. 1980), BaMV exhibited a preference for replication and accumulation in older leaves of $N$. benthamiana (Fig. 8A). In this study, we analyzed the host factors associated with BaMV-Rep protein and provided evidence that a chloroplast chaperone protein, NbcpHsp70-2, is required for the efficient replication of BaMV in older leaves of $N$. benthamiana. We found that two key amino acids in the transit peptide of NbcpHsp70-2 were crucial for both the import of NbcpHsp70-2 into older chloroplasts and the age-dependent tissue preference of BaMV.

A general feature of (+)RNA viruses is recruitment of the organelle membrane for replication (Laliberté and Sanfaçon 2010). Several lines of evidence support that BaMV replication occurs in chloroplasts. BaMV RNA interacts with a chloroplast protein, PGK, and takes advantage of its transit peptide to move from the cytoplasm to chloroplasts for replication (Cheng et al. 2013). In situ hybridization and immunogold-labeling experiments revealed BaMV gRNA in chloroplasts of BaMV-infected cells (Lin et al. 1993). In addition, the MS2 RNA tagging assay revealed BaMV gRNA in chloroplasts of $N$. benthamiana cells (Cheng et al. 2013). Furthermore, BaMV minus-strand RNA, representing the intermediate of the replication process, was detected in chloroplasts (Cheng et al. 2013).

In the present study, we provide further evidence that a nuclear-encoded and chloroplast-targeted chaperone protein, NbcpHsp70, was immunopurified with the BaMV-Rep and involved in BaMV efficient accumulation in plants (Figs. 2 and $3)$. Downregulating the expression of a specific cpHsp70 isoform, cpHsp70-2, reduced the BaMV RNA accumulation in
$N$. benthamiana protoplasts (Fig. 4). Overexpression of the mislocalized mutant $\Delta$ TPcpHsp70-2 reduced the BaMV RNA accumulation in $N$. benthamiana plants (Fig. 5), which suggests that this mutant might sequester the BaMV-Rep in the cytoplasm and hinder the assembly of replication complexes with chloroplasts. The cpHsp70 proteins play an important role in protein transportation into the chloroplast stroma $(\mathrm{Su}$ and $\mathrm{Li}$ 2010), and the mislocalized cpHsp70-2 might disrupt the chloroplast importation pathway for other essential host factors for BaMV replication such as PGK.

Other plant (+)RNA viruses such as Turnip mosaic virus (TuMV) and Turnip yellow mosaic virus (TYMV) form VRC in association with host chloroplasts (Prod'homme et al. 2003; Wei et al. 2010). The TuMV Rep protein $6 \mathrm{~K} 2$ induces the formation of vesicles at the endoplasmic reticulum membrane and then fuses to the chloroplast outer membrane for robust viral genome replication (Wei et al. 2010). The fusion of $6 \mathrm{~K} 2$ vesicles to chloroplasts is aided by the host membrane fusion protein Syp71 that is recruited to the 6K2-induced chloroplast clumping structure (Wei et al. 2013). Clumping of chloroplasts has also been observed in TYMV-infected cells (Jakubiec et al. 2004; Prod'homme et al. 2003). However, targeting the $66 \mathrm{~K}$ Rep to and assembly of the TYMV replication complexes on the chloroplast envelope appeared to solely depend on the $140 \mathrm{~K}$ Rep (Jakubiec et al. 2004). These studies indicate that viruses may use various strategies to target the chloroplast membrane for replication.

Similar to AtcpHsp70, two putative stromal Hsp70 in $N$. benthamiana were revealed by a survey of the transcriptomic database. The result showed that there were eight- and threefold increases in the expression levels of NbcpHsp70-1 and NbcpHsp70-2, respectively, at the seventh day after BaMV infection. The observation revealed the possible involvement of NbcpHsp70-1 and NbcpHsp70-2 in BaMV infection cycles. For NbcpHsp70-1, the increase might be related to the inducible host responses to BaMV-infection, because the basal level of NbcpHsp70-1 is quite low. On the other hand, the apparent lower increase of NbcpHsp70-2 expression level might be due to the higher basal expression level, which might increase the chance of being utilized by BaMV at the early stages of infection. Although the silencing of NbcpHsp70-1 and NbcpHsp70-2 affected plant growth (Fig. 3A), we demonstrated that the effect of NbcpHsp70-2 on BaMV accumulation did not result from growth defects (Fig. 4). The effect is more likely related to the chloroplast importation functions of NbcpHsp70-2. Indeed, we showed that alteration of the two key positive-charged amino acids in the transit peptide, similar to those reported for AtcpHsp70-1 of A. thaliana (Teng et al. 2012), reduced the entry efficiency of NbcpHsp70-2 into chloroplasts in older leaves and decreased BaMV accumulation (Fig. 7). This result supported the idea that viruses may take advantage of available host regulation mechanisms to enhance their fitness in the environment. To provide further evidence linking the chloroplast importation of NbcpHsp70-2 to the age-dependent tissue preference of BaMV, we replaced the NbcpHsp70-2 transit peptide with one for importation to young chloroplasts and found that the mutant could enhance BaMV accumulation in younger leaves (Fig. 8). The result demonstrated the crucial role of the transit peptide of NbcpHsp70-2 in BaMV accumulation. The observation also reinforced a possible biotechnological application for BaMV as a vector: by engineering the transit peptides of NbcpHsp70-2, we could manipulate the subcellular localization of the target protein expressed by BaMVbased viral vectors.

According to sequence comparisons, we show here that NbcpHsp70-2 is more similar to AtcpHsp70-1. AtcpHsp70-1 interacts with AbMV MP and is required for viral transport but not viral DNA accumulation (Krenz et al. 2010). Here, we 
provide the first finding of a regulatory role for NbcpHsp70 involved in replication of a $(+)$ RNA plant virus.

In addition to $\mathrm{cpHsp} 70$, several cellular chaperones, demonstrated to be involved in positively regulating BaMV replication (Huang et al. 2012b), were also detected in the immunopurified complexes by using BaMV-Rep as the bait (Fig. 2). The result implied that these molecular chaperones are present in the BaMV VRC. Cytosolic Hsp90 and Hsp70 are the most abundant molecular chaperones that play various roles in RNA virus replication, mainly via correct folding and activation of viral proteins (Verchot 2012). In BaMV replication, Hsp90 is involved in recruiting the BaMV RNA template and assembly of BaMV VRC with two distinct functional domains to interact with BaMV RNA and BaMV RdRp, respectively (Huang et al. 2012b). It has been shown that cytosolic Hsp70 and Hsp90 form multichaperone complexes together with cochaperones for their molecular chaperone function (Hahn et al. 2011). Therefore, they are often present together in the protein complexes. Moreover, Hsp70 and Hsp90 have been reported to be associated with an Hsp90 cochaperone, Hsp70/Hsp90 organizing protein, to bind chloroplast precursor proteins in cytoplasm, thereby preventing the aggregation of nonnative polypeptides and keeping them in a competent state for transferring to the chloroplast surface (Fellerer et al. 2011). Thus, the Hsp70/Hsp90 multichaperone complex may function in association with cpHsp70-2 in targeting BaMV-Rep/RNA complex to mature chloroplasts in older leaves for replication. Several reports have pointed out that Hsp90 and Hsp70 can function together to promote viral replication (Mine et al. 2012; Stahl et al. 2007; Weeks and Miller 2008), although they might assist virus replication at different stages of the infection cycles. Therefore, the precise roles for these molecular chaperones in BaMV replication should be determined mechanistically in future research. The current study revealed the ability of BaMV to exploit subcellular transport machineries for replication, and established the foundation for the future investigation of the roles of elements in such machinery.

\section{MATERIALS AND METHODS}

\section{Plant materials and growing conditions.}

Plants materials of $N$. benthamiana were grown at $28^{\circ} \mathrm{C}$ under cycles of $16 \mathrm{~h}$ of light and $8 \mathrm{~h}$ of darkness. The third or fourth leaf from the bottom to top along the stem of seedlings of the 30-day-old $N$. benthamiana was designated as "older leaf" and the sixth leaf as "younger leaf".

\section{Construction of BaMV infectious clone with an HA-tag at the C-terminal end of replicase.}

To construct pKB-RepHA, we used a two-step procedure. In the first step, construction of pCB-RepHA, a megaprimer, was synthesized by a first PCR amplification with $\mathrm{pCB}$, an infectious clone of BaMV-S (Lin et al. 2004), used as the template, with the primer pairs for ORF1HAORF2 (5'-GTTTATTCTCTAGTTAT CCATACGACGTTCCAGATTACGCATAAGTAACCTATTAGT TTAAC-3') and Ba5361R (5'-GGGCAGATGCTGTTGAAG-3'). The purified megaprimer was used in the second PCR amplification using $\mathrm{pCB}$ as template, with forward primer $\mathrm{Ba3572} F\left(5^{\prime}-\right.$ ATGAAAGCAAGGCACCATG-3'). The products of the second PCR were gel purified, digested with AfIII and NsiI, and used to replace the corresponding fragment within the $\mathrm{pCB}$ plasmid after digestion with the same restriction enzymes to generate the pCB-RepHA. The second step was to introduce the BaMVRepHA into the Agrobacterium tumefaciens binary vector, $\mathrm{pKn}$ (Prasanth et al. 2011). Plasmid pCB-RepHA was digested with $S b f I$ and $S a c I$, then cloned into pKn vector to generate the plasmid pKB-RepHA. Constructing the plasmid pKB-RepHA21 involved a similar procedure. The megaprimer was amplified by
PCR, with pCB-RepHA used as a template, with the primer pairs SGP1-21 (5'-ACTAATAGGTTACTTAACTAGAGAATAAACC TTCTTATGCGTAATCTG-3') and Ba3572F. The purified megaprimer was used in the second PCR amplification, with pCB used as a template, with a reverse primer for Ba5361R. PCR products were gel purified, digested with $A f l I I$ and NsiI, and used to replace the corresponding fragment within the $\mathrm{pCB}$ plasmid to generate the pCB-RepHA21. Plasmid pCB-RepHA21 was digested with $S b f \mathrm{I}$ and $S a c \mathrm{I}$, then cloned into $\mathrm{pKn}$ vector to generate the plasmid pKB-RepHA21.

\section{Plant inoculation by agroinfiltration.}

Plasmids pKB (Liou et al. 2014), pKB-RepHA, pKBRepHA21, and pKBG (Prasanth et al. 2011) were introduced into A. tumefaciens strain GV3850 individually by electroporation. A. tumefaciens cultures were collected by centrifugation and resuspended in infiltration buffer $(10 \mathrm{mM} 2-(N$-morpholino $)-$ ethanesulfonic acid hydrate (MES) buffer [pH 5.5] and $10 \mathrm{mM}$ $\mathrm{MgCl}_{2}$ ); suspensions were adjusted to an optical density at $600 \mathrm{~nm}$ $\left(\mathrm{OD}_{600}\right)=0.1$ and infiltrated by syringe into leaves of each test plant.

\section{RNA analysis by Northern blot hybridization.}

Total RNA were extracted from inoculated leaves or protoplasts with use of TriPure Isolation Reagent (Roche Life Science). RNA samples were separated by electrophoresis after denaturation in the presence of glyoxal and transferred to nylon membranes (Amersham) for Northern blot analysis (Lin et al. 1992). Blots were hybridized with a riboprobe specific for BaMV 3'UTR. The ${ }^{32} \mathrm{P}$-labeled probe was transcribed from HindIII-linearized pBaHB by using SP6 RNA polymerase (Lin et al. 1993).

\section{Protein analysis and antibodies.}

Total proteins were extracted from leaf tissue, and the protein samples (corresponding to $2 \mathrm{mg}$ fresh weight) were separated on 12 or $10 \%$ polyacrylamide gels containing $1 \%$ sodium dodecyl sulfate (sodium dodecyl sulfate polyacrylamide gel electrophoresis). Separated proteins were stained with Coomassie blue or detected by Western blot analysis with specific antibodies. For Western blot analysis, separated proteins were transferred to polyvinylidene difluoride membranes (Millipore) and incubated with antibodies for BaMV-TGBp1 (Chang et al. 1997), BaMV-CP (Lin et al. 1992), or monoclonal antibody for His (AbD Serotec) at 1/5,000 dilution.

\section{BaMV RdRp preparation and RdRp assay.}

Methods for preparing BaMV RdRp were as described (Huang et al. 2008), with slight modifications. Approximately $10 \mathrm{~g}$ of leaves infiltrated with A. tumefaciens carrying $\mathrm{pKn}$ or pKB-RepHA21 were harvested at the fourth day postinfiltration and frozen quickly in liquid nitrogen. Frozen leaves were homogenized at $4{ }^{\circ} \mathrm{C}$ in $20 \mathrm{ml}$ of buffer A $(50 \mathrm{mM}$ Tris- $\mathrm{HCl}[\mathrm{pH}$ 7.6], $15 \mathrm{mM} \mathrm{MgCl} 2,120 \mathrm{mM} \mathrm{KCl}, 0.1 \% \beta-\mathrm{ME}, 20 \%$ glycerol, $0.1 \mathrm{mM}$ phenylmethanesulfonyl fluoride, and protease inhibitor cocktail) (Roche Life Science), then filtered through two layers of Miracloth (CalBiochem) and centrifuged at $500 \times g$ for $10 \mathrm{~min}$ at $4^{\circ} \mathrm{C}$. The supernatant was further centrifuged at $30,000 \times g$ for $35 \mathrm{~min}$ at $4^{\circ} \mathrm{C}$. The pellet (P30) was resuspended in $4 \mathrm{ml}$ of buffer B (50 mM Tris- $\mathrm{HCl}$ [pH 8.2], $10 \mathrm{mM} \mathrm{MgCl}_{2}$, $1 \mathrm{mM}$ dithiothreitol [DTT], and protease inhibitor cocktail), stirred at $4^{\circ} \mathrm{C}$ for $1 \mathrm{~h}$, loaded onto 20 to $60 \%$ sucrose gradient in buffer $\mathrm{C}(50 \mathrm{mM}$ Tris- $\mathrm{HCl}$ [pH 7.5], $100 \mathrm{mM} \mathrm{NaCl}, 3 \mathrm{mM}$ $\mathrm{MgCl}_{2}$, and $2 \mathrm{mM} \mathrm{DTT}$ ), and centrifuged at $72,100 \times \mathrm{g}$ at $4^{\circ} \mathrm{C}$ for $7.2 \mathrm{~h}$. After sucrose density gradient centrifugation, 10 fractions were collected from the top to bottom. Fractions 5 to 7 , containing the major endogenous RdRp activities, were collected and stirred gently at $4^{\circ} \mathrm{C}$ for $1 \mathrm{~h}$ with $0.5 \%$ sarkosyl to solubilize the membrane-bound RdRp. The solubilized RdRp complexes 
(F567 $7_{\text {sark }}$ ) were centrifuged at $100,000 \times g$ for $35 \mathrm{~min}$ at $4^{\circ} \mathrm{C}$ and the supernatant was collected (S100) for immunoprecipitation assay. The RdRp activity assay with endogenous RNA templates involved $20 \mu \mathrm{l}$ of $\mathrm{F} 567_{\text {sark }}$ or S100, as described (Cheng et al. 2001).

\section{Immunoprecipitation.}

To identify the host proteins associated with BaMV replicase complexes, immunoprecipitation was performed with anti-HA antibody (H6958; purchased from Sigma-Aldrich). Before immunoprecipitation of the BaMV replicase complexes, $1 \mathrm{ml}$ of S100 was incubated with $0.1 \mathrm{ml}$ of $50 \%$ (vol/ $/ \mathrm{vol}$ ) Protein ASepharose CL-4B suspension (GE Healthcare Life Sciences) for $1.5 \mathrm{~h}$ and centrifuged at $3,000 \times g$ for $2 \mathrm{~min}$ at $4^{\circ} \mathrm{C}$ to remove protein components interacting nonspecifically with Protein A. The S100 precleaned with Protein A was designated "input". To immunoprecipitate the BaMV-Rep complexes, $0.5 \mathrm{ml}$ of input was mixed with $5 \mu \mathrm{l}$ of anti-HA antibody on a rotamixer for $4 \mathrm{~h}$ at $4^{\circ} \mathrm{C}$. Proteins interacting with Rep-HA were precipitated by the addition of $20 \mu \mathrm{l}$ of Protein A magnetic beads (GE Healthcare), followed by incubation for $2 \mathrm{~h}$ at $4^{\circ} \mathrm{C}$. After washing and elution, the samples underwent Western blot analyses with antibodies specific to HA (H6908; Sigma-Aldrich), BaMV CP, Hsp70 (Abcam), Hsp90, or cpHsp70 (Agrisera).

\section{VIGS.}

A 291-bp fragment of the cpHsp70 coding sequence from $N$. benthamiana was amplified by RT-PCR by using Moloney murine leukemia virus (M-MLV) reverse transcription (Promega Corp.), oligo dT, and gene-specific primers (XbaI/NbcpHsp70 VIGS-F: 5'-GCTCTAGAGCTTCCGGCTTGATGG-3' and BamHI/NbcpHsp70 VIGS-R: 5'-GCGGATCCGTTCTTTCAG CTGC-3'). PCR products were gel purified, digested with XbaI and BamHI, and cloned into the pTRV2 plasmid (Ratcliff et al. 2001) after digestion with the corresponding restriction enzymes to generate pTRV2-cpHsp70. Full-length cpHsp70-1 and cpHs70-2 sequences from $N$. benthamiana were amplified by RT-PCR by using M-MLV reverse transcription (Promega Corp.), oligo $\mathrm{dT}_{(18)}$, and gene-specific primers for NbcpHsp70-1 (XbaI/cpHsp70-1[+]1: 5'-GCTCTAGAATGAGCTCAATTATG GCTG-3') and NbcpHsp70-2 (XbaI/cpHsp70-2[+]1: 5' -GCTCT AGAATGGCGTCTTCAACAGCTC-3'). The PCR products were cloned into pyT\&A (Yeastern) to generate pyTAcpHsp70-1 and pyTAcpHsp70-2, respectively. To prepare VIGS constructs for specific silencing of NbcpHsp70-1 and NbcpHsp70-2, the plasmids pTRV2/cpHsp70-1/3'UTR and pTRV2/cpHsp702/3'UTR were generated. The 3'UTR of NbcpHsp70-1 and NbcpHsp70-2 were amplified by PCR from pyTAcpHsp70-1 and pyTAcpHsp70-2 templates with primer pairs: for NbcpHsp70-1, XbaI/NbcpHsp70-1/3'UTR(+)1 (5'-GCTCTAGAGAAACAGA ATCAAGAATTC-3') and BamHI/NbcpHsp70-1/3'UTR(-)194 (5'-GCGGATCCCATACTGGTTTGGAAAATC-3'); and, for NbcpHsp70-2, XbaI/NbcpHsp70-2/3'UTR(+)1 (5'-GCTCTA GAGCATAGAAGAGCAATTTTG-3') and BamHI/NbcpHsp702/3'UTR(-)201 (5'-GCGGATCCCATCTTGCAAGCAAGAG-3'). PCR products were gel purified, digested with $X b a \mathrm{I}$ and BamHI, and cloned into pTRV2 plasmid to generate the pTRV2/cpHsp70-1/3'UTR and pTRV2/cpHsp70-2/3'UTR, respectively. pTRV1- and pTRV2-based constructs in A. tumefaciens (C58C1) were grown and infiltrated into $N$. benthamiana leaves as described (Huang et al. 2012b). Gene silencing was confirmed by standard RNA extraction and quantitative RT-PCR analysis.

\section{Real-time PCR.}

Total RNA from leaf tissues was extracted with TriPure Isolation Reagent (Roche Life Science). First-strand cDNA was synthesized by using an oligo $\mathrm{dT}_{(18)}$ primer and M-MLV HP reverse transcription (Epicentre). Twofold diluted cDNA $(2 \mu \mathrm{l})$ gene-specific primers (cpHsp70 RT-PCR-5' : 5'-GGCAGCTCT TAACCAAGAAG-3' and cpHsp70 RT-PCR-3': 5'-GGCT GAGGATGATGTGTC-3'), and the SYBR qPCR master mix (LabStar) were used for quantitative RT-PCR analyses of NbcpHsp70 mRNA level with a TOptical Gradient 96 RealTime PCR Thermal Cycler (Biometra). To normalize the mRNA levels of target genes between samples, relative actin mRNA levels were determined with specific primers (Actin-F: 5'-GATGAAGATACTCACAGAAAGA-3' and Actin-R: 5'GTGGTTTCATGAATGCCAGCA-3'). For semiquantitative RT-PCR, the first-strand cDNA from samples were used for PCR with the sets of primers to amplify NbHsp70 isoforms.

\section{Protoplast isolation and viral RNA infection.}

The protoplasts were generated from the Luc-, PDS-, NbcpHsp70-1-, or NbcpHsp70-2-knockdown plants. Methods for preparing and isolating protoplasts were described (Huang et al. 2012b).

\section{Transient expression}

of NbcpHsp70-2 and NbcpHsp70-2 mutants.

Plasmid construction for transient expression of NbcpHsp702 and NbcpHsp70-2 mutants are described in Supplementary Method S1. Plasmids for transient expression of NbcpHsp70-2 and NbcpHsp70-2 mutants were introduced into A. tumefaciens strain GV3850 individually by electroporation. A. tumefaciens cultures were collected by centrifugation and resuspended in infiltration buffer. Suspensions were adjusted to $\mathrm{OD}_{600}=0.5$ and infiltrated by syringe into leaves of each test plant.

Confocal microscopy. To visualize the localization of NbcpHsp70-2 and NbcpHsp70-2 mutants in N. benthamiana protoplasts or leaf tissues, plasmids pEpcpHsp70-2-OFP, pEp $\Delta$ TPcpHsp70-2-OFP, and pEpRK2425EE-OFP were generated. The coding sequences of cpHsp70-2 and $\Delta$ TPcpHsp70-2 were amplified by PCR with pyTAcpHsp70-2 as the template

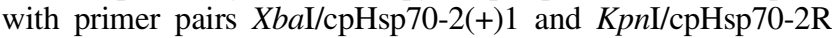
(5'-GGGGTACCCTTGCTCTCGGTG-3') for cpHsp70-2 and

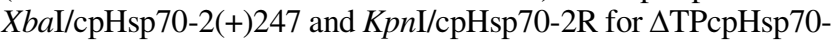
2. PCR products were gel purified, digested with $X b a \mathrm{I}$ and $K p n \mathrm{I}$, and used to replace the corresponding fragment within pEpyonOFP after digestion with the same restriction enzymes to generate the pEpcpHsp70-2-OFP and $\mathrm{pEp} \Delta \mathrm{TPcpHsp70-2-OFP,} \mathrm{respec-}$ tively. To construct pEpRK2425EE-OFP, PCR was performed with pEpRK2425EE as the template with the primer pairs for

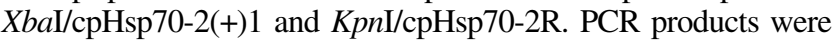
gel purified, digested with $X b a \mathrm{I}$ and $K p n \mathrm{I}$, and used to replace the corresponding fragment within pEpyon-OFP to generate pEpRK2425EE-OFP. To construct pEpRbcS-cpHsp70-2-OFP, PCR was performed with pBIN-rbcs-EF1a-T7 (Cheng et al. 2013 ) as the template with the primer pairs $X b a \mathrm{I} / \mathrm{rbcS} / \mathrm{F}$ (5'-GTCT AGAATGGCTTCCTCTGTTCTTTCCT-3') and $X b a \mathrm{I} / \mathrm{rbcSTP/R}$ (5'-GTCTAGATTGGACTCTTCCACCATTGC-3'). PCR products were gel purified, digested with $X b a \mathrm{I}$, and used to replace the corresponding fragment within $\mathrm{pEp} \triangle \mathrm{TPcpHsp} 70-2-\mathrm{OFP}$ to gener-

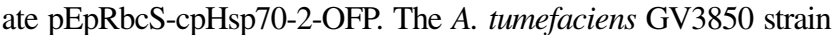
containing pEpyon-OFP, pEpcpHsp70-2-OFP, pEp $\Delta$ TPcpHsp70-2OFP, pEpRK2425EE-OFP, or pEpRbcS-cpHsp70-2-OFP was infiltrated into $N$. benthamiana leaves. Three days later, images of protoplasts or leaf tissues were obtained under an inverted fluorescence confocal microscope (FV1000; Olympus) with 543- and 633-nm laser excitation for OFP and chloroplast autofluorescence imaging, respectively.

\section{GFP pull-down assay.}

To verify the interactions between BaMV-Rep and NbcpHsp702, A. tumefaciens strain GV3850 containing pEpyon-GFP, 
pEpRep-GFP, pEpcpHsp70-2-OFP, or pEp $\Delta$ TPcpHsp70-2-OFP was infiltrated into $N$. benthamiana for in planta assays. To construct $\mathrm{pEpRep-GFP,} \mathrm{PCR} \mathrm{was} \mathrm{performed} \mathrm{with} \mathrm{pCB}$ as the template with the primer pairs PstI/ORF1F (5'-AACTGCA GATGGCACTCGTTTCTAAAG- $\left.3^{\prime}\right)$ and $\mathrm{XbaI} / \mathrm{ORF} 1 \mathrm{R}\left(5^{\prime}\right.$-GCT CTAGAACTAGAGAATAAACCTTC-3'). PCR products were gel purified, digested with PstI and $X b a \mathrm{I}$, then cloned into pEpyon-GFP to generate pEpRep-GFP. After coexpression of combinations for 2 dpai, whole proteins were extracted from infiltrated leaves with three volumes of binding buffer $(20 \mathrm{mM}$ Tris-HCl [pH7.5], $2 \mathrm{mM} \mathrm{MgCl}_{2}, 300 \mathrm{mM} \mathrm{NaCl}$, and $5 \mathrm{mM}$ DTT) containing protease inhibitor cocktail. The total proteins were centrifuged at $4,000 \times g$ for $10 \mathrm{~min}$ at $4^{\circ} \mathrm{C}$ and the supernatant was transferred to a precooled tube as input. To pull down the BaMV-Rep-GFP binding complexes, $0.5 \mathrm{ml}$ of input was mixed with $10 \mu$ l of GFP-Trap_M beads (ChromoTek) on a rotamixer for $2 \mathrm{~h}$ at $4^{\circ} \mathrm{C}$. After washing and elution, proteins interacting with Rep-GFP underwent Western blot analysis with antibodies specific to OFP.

\section{ACKNOWLEDGMENTS}

We thank C. H. Yang at National Chung Hsing University for kindly providing plasmids pEpyon-GFP and pEpyon-OFP. This research was supported by the Ministry of Science and Technology, R.O.C. (MOST-1032321-B-005-023-MY2 and NSC-101-2313-B-005-036-MY3).

\section{LITERATURE CITED}

Alam, S. B., and Rochon, D. 2016. Cucumber necrosis virus recruits cellular heat shock protein 70 homologs at several stages of infection. J. Virol. 90:3302-3317.

Carella, P., Wilson, D. C., and Cameron, R. K. 2015. Some things get better with age: Differences in salicylic acid accumulation and defense signaling in young and mature Arabidopsis. Front. Plant Sci. 5:775.

Chang, B. Y., Lin, N. S., Liou, D. Y., Chen, J. P., Liou, G. G., and Hsu, Y. H. 1997. Subcellular localization of the $28 \mathrm{kDa}$ protein of the triple-geneblock of bamboo mosaic potexvirus. J. Gen. Virol. 78:1175-1179.

Cheng, J. H., Ding, M. P., Hsu, Y. H., and Tsai, C. H. 2001. The partial purified RNA-dependent RNA polymerases from bamboo mosaic potexvirus and potato virus $\mathrm{X}$ infected plants containing the templatedependent activities. Virus Res. 80:41-52.

Cheng, S. F., Huang, Y. P., Chen, L. H., Hsu, Y. H., and Tsai, C. H. 2013. Chloroplast phosphoglycerate kinase is involved in the targeting of Bamboo mosaic virus to chloroplasts in Nicotiana benthamiana plants. Plant Physiol. 163:1598-1608.

Collum, T. D., Padmanabhan, M. S., Hsieh, Y. C., and Culver, J. N. 2016. Tobacco mosaic virus-directed reprogramming of auxin/indole acetic acid protein transcriptional responses enhances virus phloem loading. Proc. Natl. Acad. Sci. USA 113:E2740-E2749.

Develey-Rivière, M. P., and Galiana, E. 2007. Resistance to pathogens and host developmental stage: A multifaceted relationship within the plant kingdom. New Phytol. 175:405-416.

Dufresne, P. J., Thivierge, K., Cotton, S., Beauchemin, C., Ide, C., Ubalijoro, E., Laliberté, J. F., and Fortin, M. G. 2008. Heat shock 70 protein interaction with Turnip mosaic virus RNA-dependent RNA polymerase within virus-induced membrane vesicles. Virology 374 : 217-227.

Fellerer, C., Schweiger, R., Schöngruber, K., Soll, J., and Schwenkert, S. 2011. Cytosolic HSP90 cochaperones HOP and FKBP interact with freshly synthesized chloroplast preproteins of Arabidopsis. Mol. Plant 4: 1133-1145.

Griffin, D. E. 2016. Alphavirus encephalomyelitis: Mechanisms and approaches to prevention of neuronal damage. Neurotherapeutics 13: 455-460.

Hahn, A., Bublak, D., Schleiff, E., and Scharf, K. D. 2011. Crosstalk between Hsp90 and Hsp70 chaperones and heat stress transcription factors in tomato. Plant Cell 23:741-755.

Hirsch, M. S., Zisman, B., and Allison, A. C. 1970. Macrophages and agedependent resistance to Herpes simplex virus in mice. J. Immunol. 104 1160-1165.

Huang, Y. W., Hu, C. C., Lin, N. S., and Hsu, Y. H. 2012a. Unusual roles of host metabolic enzymes and housekeeping proteins in plant virus replication. Curr. Opin. Virol. 2:676-682.
Huang, Y. W., Hu, C. C., Lin, N. S., Tsai, C. H., and Hsu, Y. H. 2008. In vitro replication of Bamboo mosaic virus satellite RNA. Virus Res. 136: 98-106.

Huang, Y. W., Hu, C. C., Liou, M. R., Chang, B. Y., Tsai, C. H., Meng, M., Lin, N. S., and Hsu, Y. H. 2012b. Hsp90 interacts specifically with viral RNA and differentially regulates replication initiation of Bamboo mosaic virus and associated satellite RNA. PLoS Pathog. 8:e1002726.

Hyodo, K., and Okuno, T. 2014. Host factors used by positive-strand RNA plant viruses for genome replication. J. Gen. Plant Pathol. 80:123-135.

Jakubiec, A., Notaise, J., Tournier, V., Héricourt, F., Block, M. A., Drugeon, G., van Aelst, L., and Jupin, I. 2004. Assembly of Turnip yellow mosaic virus replication complexes: Interaction between the proteinase and polymerase domains of the replication proteins. J. Virol. 78:7945-7957.

Kim, K. H., and Hemenway, C. 1997. Mutations that alter a conserved element upstream of the potato virus $\mathrm{X}$ triple block and coat protein genes affect subgenomic RNA accumulation. Virology 232:187-197.

Krenz, B., Windeisen, V., Wege, C., Jeske, H., and Kleinow, T. 2010. A plastid-targeted heat shock cognate $70 \mathrm{kDa}$ protein interacts with the Abutilon mosaic virus movement protein. Virology 401:6-17.

Kus, J. V., Zaton, K., Sarkar, R., and Cameron, R. K. 2002. Age-related resistance in Arabidopsis is a developmentally regulated defense response to Pseudomonas syringae. Plant Cell 14:479-490.

Labrada, L., Liang, X. H., Zheng, W., Johnston, C., and Levine, B. 2002. Age-dependent resistance to lethal alphavirus encephalitis in mice: Analysis of gene expression in the central nervous system and identification of a novel interferon-inducible protective gene, mouse ISG12. J. Virol. 76:11688-11703.

Laliberté, J. F., and Sanfaçon, H. 2010. Cellular remodeling during plant virus infection. Annu. Rev. Phytopathol. 48:69-91.

Lee, C. C., Ho, Y. N., Hu, R. H., Yen, Y. T., Wang, Z. C., Lee, Y. C., Hsu, Y. H., and Meng, M. 2011. The interaction between Bamboo mosaic virus replication protein and coat protein is critical for virus movement in plant hosts. J. Virol. 85:12022-12031.

Lee, Y. S., Hsu, Y. H., and Lin, N. S. 2000. Generation of subgenomic RNA directed by a satellite RNA associated with bamboo mosaic potexvirus: Analyses of potexvirus subgenomic RNA promoter. J. Virol. 74: 10341-10348.

Lin, B. L., Wang, J. S., Liu, H. C., Chen, R. W., Meyer, Y., Barakat, A., and Delseny, M. 2001. Genomic analysis of the Hsp70 superfamily in Arabidopsis thaliana. Cell Stress Chaperones 6:201-208.

Lin, J. W., Ding, M. P., Hsu, Y. H., and Tsai, C. H. 2007. Chloroplast phosphoglycerate kinase, a gluconeogenetic enzyme, is required for efficient accumulation of Bamboo mosaic virus. Nucleic Acids Res. 35: 424-432.

Lin, M. K., Chang, B. Y., Liao, J. T., Lin, N. S., and Hsu, Y. H. 2004. Arg-16 and Arg-21 in the N-terminal region of the triple-gene-block protein 1 of Bamboo mosaic virus are essential for virus movement. J. Gen. Virol. 85: 251-259.

Lin, N. S., Chen, C. C., and Hsu, Y. H. 1993. Post-embedding in situ hybridization for localization of viral nucleic acid in ultra-thin sections. J. Histochem. Cytochem. 41:1513-1519.

Lin, N. S., Lin, B. Y., Lo, N. W., Hu, C. C., Chow, T. Y., and Hsu, Y. H 1994. Nucleotide sequence of the genomic RNA of bamboo mosaic potexvirus. J. Gen. Virol. 75:2513-2518.

Lin, N. S., Lin, F. Z., Huang, T. Y., and Hsu, Y. H. 1992. Genome properties of bamboo mosaic virus. Phytopathology 82:731-734.

Liou, M. R., Huang, Y. W., Hu, C. C., Lin, N. S., and Hsu, Y. H. 2014. A dual gene-silencing vector system for monocot and dicot plants. Plant Biotechnol. J. 12:330-343.

Mayer, M. P. 2005. Recruitment of Hsp70 chaperones: A crucial part of viral survival strategies. Rev. Physiol. Biochem. Pharmacol. 153:1-46.

Mine, A., Hyodo, K., Tajima, Y., Kusumanegara, K., Taniguchi, T., Kaido, M., Mise, K., Taniguchi, H., and Okuno, T. 2012. Differential roles of Hsp70 and Hsp90 in the assembly of the replicase complex of a positivestrand RNA plant virus. J. Virol. 86:12091-12104.

Mine, A., Takeda, A., Taniguchi, T., Taniguchi, H., Kaido, M., Mise, K., and Okuno, T. 2010. Identification and characterization of the 480kilodalton template-specific RNA-dependent RNA polymerase complex of Red clover necrotic mosaic virus. J. Virol. 84:6070-6081.

Nagy, P. D., and Pogany, J. 2012. The dependence of viral RNA replication on co-opted host factors. Nat. Rev. Microbiol. 10:137-149.

Nagy, P. D., Pogany, J., and Xu, K. 2016. Cell-free and cell-based approaches to explore the roles of host membranes and lipids in the formation of viral replication compartment induced by tombusviruses. Viruses 8:68

Nagy, P. D., Wang, R. Y., Pogany, J., Hafren, A., and Makinen, K. 2011. Emerging picture of host chaperone and cyclophilin roles in RNA virus replication. Virology 411:374-382. 
Nakasugi, K., Crowhurst, R. N., Bally, J., Wood, C. C., Hellens, R. P., and Waterhouse, P. M. 2013. De novo transcriptome sequence assembly and analysis of RNA silencing genes of Nicotiana benthamiana. PLoS One 8:e59534.

Nishikiori, M., Dohi, K., Mori, M., Meshi, T., Naito, S., and Ishikawa, M 2006. Membrane-bound tomato mosaic virus replication proteins participate in RNA synthesis and are associated with host proteins in a pattern distinct from those that are not membrane bound. J. Virol. 80:8459-8468.

Park, C. J., and Seo, Y. S. 2015. Heat shock proteins: A review of the molecular chaperones for plant immunity. Plant Pathol. J. 31:323-333.

Pogany, J., Stork, J., Li, Z., and Nagy, P. D. 2008. In vitro assembly of the Tomato bushy stunt virus replicase requires the host Heat shock protein 70. Proc. Natl. Acad. Sci. USA 105:19956-19961.

Prasanth, K. R., Huang, Y. W., Liou, M. R., Wang, R. Y., Hu, C. C., Tsai, C. H., Meng, M., Lin, N. S., and Hsu, Y. H. 2011. Glyceraldehyde 3phosphate dehydrogenase negatively regulates the replication of Bamboo mosaic virus and its associated satellite RNA. J. Virol. 85:8829-8840.

Prod'homme, D., Jakubiec, A., Tournier, V., Drugeon, G., and Jupin, I. 2003. Targeting of the Turnip yellow mosaic virus $66 \mathrm{~K}$ replication protein to the chloroplast envelope is mediated by the $140 \mathrm{~K}$ protein. J. Virol. 77:9124-9135.

Ratcliff, F., Martin-Hernandez, A. M., and Baulcombe, D. C. 2001. Technical advance: Tobacco rattle virus as a vector for analysis of gene function by silencing. Plant J. 25:237-245.

Sadeghi, S. E., Dedryver, C. A., Riault, G., and Tanguy, S. 2000. Variation in virus content among individual leaves and roots of barley and wheat infected with a BYDV-PAV isolate. J. Agric. Sci. Tech. 2:151-160.

Stahl, M., Retzlaff, M., Nassal, M., and Beck, J. 2007. Chaperone activation of the hepadnaviral reverse transcriptase for template RNA binding is established by the Hsp70 and stimulated by the Hsp90 system. Nucleic Acids Res. 35:6124-6136.

Su, P. H., and Li, H. M. 2008. Arabidopsis stromal 70-kD heat shock proteins are essential for plant development and important for thermotolerance of germinating seeds. Plant Physiol. 146:1231-1241.
Su, P. H., and Li, H. M. 2010. Stromal Hsp70 is important for protein translocation into pea and Arabidopsis chloroplasts. Plant Cell 22: 1516-1531.

Takahashi, T. 1972. Studies on viral pathogenesis in plant hosts. III. Leaf age-dependent susceptibility to tobacco mosaic virus in 'Samsun NN' and 'Samsun' tobacco plants. Phytopathol. Z. 75:140-155.

Teng, Y. S., Chan, P. T., and Li, H. M. 2012. Differential age-dependent import regulation by signal peptides. PLoS Biol. 10:e1001416.

Venekamp, J. H., and Beemster, A. B. R. 1980. Mature plant resistance of potato against some virus diseases. 1. Concurrence of development of mature plant resistance against potato virus $\mathrm{X}$, and decrease of ribosome and RNA content. Neth. J. Plant Pathol. 86:1-10.

Venekamp, J. H., Schepers, A., and Bus, C. B. 1980. Mature plant resistance of potato against some virus diseases. 3. Mature plant resistance against potato virus $\mathrm{Y}^{\mathrm{N}}$, indicated by decrease in ribosome content in aging potato plants under field conditions. Neth. J. Plant Pathol. 86:301-309.

Verchot, J. 2012. Cellular chaperones and folding enzymes are vital contributors to membrane bound replication and movement complexes during plant RNA virus infection. Front. Plant Sci. 3:275.

Weeks, S. A., and Miller, D. J. 2008. The heat shock protein 70 cochaperone $Y D J 1$ is required for efficient membrane-specific flock house virus RNA replication complex assembly and function in Saccharomyces cerevisiae. J. Virol. 82:2004-2012.

Wei, T., Huang, T. S., McNeil, J., Laliberté, J. F., Hong, J., Nelson, R. S., and Wang, A. 2010. Sequential recruitment of the endoplasmic reticulum and chloroplasts for plant potyvirus replication. J. Virol. 84:799-809.

Wei, T., Zhang, C., Hou, X., Sanfaçon, H., and Wang, A. 2013. The SNARE protein Syp71 is essential for Turnip mosaic virus infection by mediating fusion of virus-induced vesicles with chloroplasts. PLoS Pathog. 9: e1003378.

Yang, C. C., Liu, J. S., Lin, C. P., and Lin, N. S. 1997. Nucleotide sequence and phylogenetic analysis of a bamboo mosaic potexvirus isolate from common bamboo (Bambusa vulgaris McClure). Bot. Bull. Acad. Sin. 38: 77-84. 